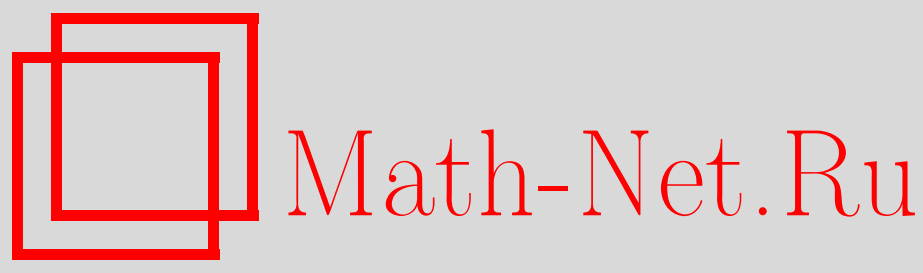

Б. А. Дубровин, Т. В. Скрыпник, Классический дубль, $R$-операторы и отрицательные потоки интегрируемых иерархий, $Т M \Phi, 2012$, том 172, номер 1, 40-63

DOI: https://doi.org/10.4213/tmf6903

Использование Общероссийского математического портала Math-Net.Ru подразумевает, что вы прочитали и согласны с пользовательским соглашением http://www . mathnet.ru/rus/agreement

Параметры загрузки:

IP : 54.164 .48 .24

26 апреля 2023 г., 16:19:17

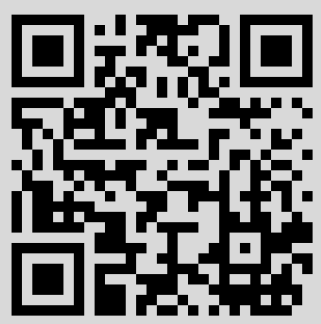




\title{
ФИЗИКА
}

Том 172, № 1

июль, 2012

(C) 2012 г. $\quad$ Б. А. Дубровин ${ }^{* \dagger}$, Т. В. Скрыпник ${ }^{\dagger \ddagger \S}$

\section{КЛАССИЧЕСКИЙ ДУБЛЬ, R-ОПЕРАТОРЫ И ОТРИЦАТЕЛЬНЫЕ ПОТОКИ ИНТЕГРИРУЕМЫХ ИЕРАРХИЙ}

\begin{abstract}
$\mathrm{C}$ использованием классического дубля $\mathcal{G}$ алгебры Ли $\mathfrak{g}$, оснащенной классическим $R$-оператором, определены два набора функций, коммутирующих по отношению к изначальной скобке Ли-Пуассона на дуальном пространстве $\mathfrak{g}^{*}$ и его расширениях. Детально рассмотрены примеры алгебр Ли $\mathfrak{g}$ с $R$-операторами Адлера-Костанта-Симса и два соответствующих набора взаимно коммутирующих функций. На основе полученных коммутирующих гамильтоновых потоков на различных расширениях алгебры $\mathfrak{g}$ выведено уравнение нулевой кривизны с $\mathfrak{g}$-значными $U-V$-парами. Среди полученных уравнений содержатся так называемые отрицательные потоки интегрируемых иерархий. Предлагаемый подход проиллюстрирован примерами абелевых и неабелевых уравнений двумерного поля Тоды.
\end{abstract}

Ключевые слова: классические $R$-операторы, интегрируемые иерархии.

\section{1. ВВЕДЕНИЕ}

Теория иерархий интегрируемых уравнений в частных производных базируется на возможности записать каждое из уравнений иерархии с помощью так называемого представления нулевой кривизны $U_{t}-V_{x}+[U, V]=0$, где $U-V$-пара принимает значения в некоторой бесконечномерной алгебре Ли $\mathfrak{g}$ (например, в алгебре матричнозначных рядов Лорана от одной комплексной переменной $\lambda$ ). Существует несколько подходов к построению уравнений нулевой кривизны, базирующихся на алгебрах Ли. Один из них [1] основан на том, что уравнения нулевой кривизны интепретируются как одно уравнение Лакса (Эйлера-Арнольда), записанное на центрально расширенной алгебре $\mathfrak{g}$-значных функций переменной $x$. В рамках этого подхода роль одного оператора Лакса играет оператор $\partial_{x}-U$, а второй оператор в паре Лакса совпадает с оператором $V$.

*International School for Advanced Studies, Trieste, Italy. E-mail: dubrovin@sissa.it

${ }^{\dagger}$ Московский государственный университет им. М. В. Ломоносова, Москва, Россия

${ }^{\ddagger}$ Universita di Milano Bicocca, Milan, Italy. E-mail: taras.skrypnyk@unimib.it

${ }^{\S}$ Институт теоретической физики им. Н. Н. Боголюбова, Киев, Украина.

E-mail: tskrypnyk@imath.kiev.ua 
При альтернативном подходе [2], [3] уравнение нулевой кривизны интерпретируется как условие совместности двух вспомогательных уравнений Лакса (Эйлера-Арнольда). Коммутативность этих лаксовых потоков гарантируется коммутативностью соответствующих гамильтонианов относительно скобки Ли-Пуассона. При этом подходе элементы $U$ и $V$ из уравнений нулевой кривизны совпадают с алгеброзначными градиентами коммутирующих гамильтонианов, полученных с помощью схемы Адлера-Костанта-Саймса (AKC). Детальнее, эти гамильтонианы получаются путем ограничения функций Казимира алгебры $\mathfrak{g}$ на пространства, дуальные к подалгебрам $\mathfrak{g}_{ \pm}$, где $\mathfrak{g}=\mathfrak{g}_{+}+\mathfrak{g}_{-}$. Такой подход позволяет получить два типа интегрируемых уравнений, ассоциированных с алгеброй Ли $\mathfrak{g}$, а именно интегрируемые уравнения с операторами $U$ и $V$, принадлежащими той же подалгебре Ли $\mathfrak{g}_{+}$или $\mathfrak{g}_{-}$. Однако подход работы [3] не покрывает всех известных интегрируемых уравнений. В частности, он не работает для интегрируемых уравнений (иногда называемых отрицательными потоками интегрируемых иерархий $)^{1)}$, которые обладают $U-V$-парами с $U$-оператором, принадлежащим подалгебре $\mathfrak{g}_{+}$, и $V$-оператором, принадлежащим подалгебре $\mathfrak{g}_{-}$. В работе [7] уравнения такого типа включены в общую схему путем доказательства того, что ограничения функций Казимира алгебры $\mathfrak{g}$ на пространства, дуальные подалгебрам $\mathfrak{g}_{+}$и $\mathfrak{g}_{-}$, коммутируют не только внутри каждой группы, но и между группами. Это позволяет получить отрицательные потоки интегрируемых иерархий как следствие коммутативности потоков Лакса, порожденных "положительными" и "отрицательными" гамильтонианами. В работах [8], [9] предложено обобщение этой схемы на случай алгебр Ли $\mathfrak{g}$, обладающих общим классическим $R$-оператором, не всегда связанным с разложением $\mathfrak{g}=\mathfrak{g}_{+}+\mathfrak{g}_{-}$(т. е. не всегда связанным со схемой $\mathrm{AKC})$. Показано, что ограничения функций Казимира алгебры $\mathfrak{g}$ на подалгебры $\mathfrak{g}_{R_{ \pm}}$, где $\mathfrak{g}_{R_{ \pm}}=\operatorname{Im} R_{ \pm}$, коммутируют не только внутри каждой группы, но и между группами. Это наблюдение позволяет получить два набора взаимно коммутирующих функций на дуальном пространстве $\mathfrak{g}^{*}$ и три типа уравнений нулевой кривизны, в частности тот тип уравнений, который отвечает отрицательным потокам солитонных иерархий [9]. Заметим, что соответствующая коммутативность не следует из стандартной $R$-матричной схемы [10] на $\mathfrak{g}$.

Тем не менее оказалось, что схема, предложенная в работах [8], [9], все еще не является наиболее общим подходом к генерированию коммутативных потоков на $\mathfrak{g}^{*}$ и, следовательно, к конструированию солитонных иерархий с $\mathfrak{g}$-значными $U-V$-парами. В частности, предложенная схема не включает в себя бесконечнокомпонентную иерархию Тоды и не порождает соответствующие вспомогательные уравнения Лакса [11].

В настоящей работе мы предлагаем более общий подход к построению коммутирующих потоков и получаем уравнение нулевой кривизны с $\mathfrak{g}$-значными $U-V$-парами. С этой целью мы рассматриваем коммутативные потоки не на $\mathfrak{g}^{*}$, а на $\mathcal{G}^{*}$, где $\mathcal{G}$ - классический дубль алгебры $\mathfrak{g}$. Мы используем тот факт, что классический $R$-оператор на $\mathfrak{g}$ индуцирует естественный $R$-оператор на $\mathcal{G}=\mathfrak{g} \oplus \mathfrak{g}[12]$. Оказывается, что $R$-оператор $\mathcal{R}$ на $\mathcal{G}$ всегда имеет тип АKC вне зависимости от того, какой вид имел $R$-оператор на g. Из этого следует, что справедливо разложение $\mathcal{G}_{\mathcal{R}}=\mathcal{G}_{\mathcal{R}_{+}} \ominus \mathcal{G}_{\mathcal{R}_{-}}$, где алгебра $\mathcal{G}_{\mathcal{R}}-$ это линейное пространство $\mathcal{G}$, оснащенное так

1) В настоящей работе мы не можем процитировать или сделать обзор всех публикаций на эту тему. Мы отсылаем заинтересованного читателя, например, к недавним работам [4]-[6]. 
называемой $\mathcal{R}$-скобкой [10]. Более того, оказывается, что $\mathcal{G}_{\mathcal{R}_{+}} \simeq \mathfrak{g}, \mathcal{G}_{\mathcal{R}_{-}} \simeq \mathfrak{g}_{R}$, где алгебра $\mathfrak{g}_{R}$ - это линейное пространство $\mathfrak{g}$, оснащенное $R$-скобкой, и $\mathcal{G}_{\mathcal{R}_{ \pm}} \equiv \operatorname{Im} \mathcal{R}_{ \pm}[12]$.

Таким образом, нашим первым наблюдением является тот факт, что, применяя стандартную $R$-операторную схему [10] к алгебре Ли $\mathcal{G}$, которая оснащена $R$-оператором $\mathcal{R}$, можно получить набор коммутирующих потоков на расширении алгебры $\mathfrak{g}$ некоторой алгеброй Ли $\mathfrak{a}$, где $\mathfrak{a}=\mathfrak{g}_{R} / J_{R}$ и $J_{R}$ является идеалом алгебры $\mathfrak{g}_{R}$. В частном случае $J_{R}=\mathfrak{g}_{R}$ данное наблюдение позволяет получить простое доказательство результатов работы [8] о коммутативности ограничений функции Казимира алгебры $\mathfrak{g}$ на подалгебры $\mathfrak{g}_{R_{ \pm}}$. Тем самым мы показываем, что результаты работы [8] могут быть выведены путем использования обычной $R$-операторной схемы. В случае $J_{R}=\left[\mathfrak{g}_{R}, \mathfrak{g}_{R}\right]$ мы получаем важное обобщение упомянутого выше результата: доказываем коммутативность ограничения функций Казимира алгебры $\mathfrak{g}$ на подалгебры $\mathfrak{g}_{R_{ \pm}}$, сдвинутые при помощи постоянных элементов $c_{\mp} \in\left[\mathfrak{g}_{R_{\mp}}, \mathfrak{g}_{R_{\mp}}\right]$ соответственно. Заметим, что в этом случае полученные функции коммутируют относительно скобки Ли-Пуассона на алгебре $\mathfrak{g}$, “сдвинутой” постоянным элементом $c_{+}-c_{-}$.

Рассмотрение коммутативных семейств на более сложных факторах (с неабелевой алгеброй $\mathfrak{a}$ ) тоже может быть полезным в теории солитонных уравнений. Действительно, наше второе наблюдение состоит в том, что, какой бы фактор алгебры $\mathcal{G}_{\mathcal{R}}$ мы ни рассматривали, в уравнениях Лакса $\dot{L}=[L, M]$, которые соответствуют коммутирующим гамильтонианам (функциям Казимира, ограниченным на рассматриваемый фактор), можно выбрать $M$-оператор, принимающий значение в алгебре Ли $\mathfrak{g}$. Из этого следует, что можно получить уравнение нулевой кривизны с $\mathfrak{g}$-значными $U-V$-парами как условие совместности уравнений Лакса на $\mathfrak{g} \ominus \mathfrak{a}$.

Мы иллюстрируем предлагаемый метод примерами абелевого и неабелевого уравнений поля Тоды (см. работы [13]-[15] и ссылки в них), которые естественным образом получаются в рамках изложенной схемы в случае, когда $\mathfrak{g}$ является алгеброй петель в разных градуировках. Соответствующая фактор-алгебра в этом случае представляет собой простейшее неабелево расширение алгебры $\mathfrak{g}$, полученное в рамках изложенной выше конструкции. В случае алгебры $\mathfrak{g}=g l((\infty))$, которая оснащена естественным разложением в сумму двух подалгебр, совпадающих с верхнетреугольными и строго нижнетреугольными матрицами, мы вновь выводим результаты работы [16], касающиеся скобки Ли-Пуассона, а также основанной на теории алгебр Ли интерпретации бесконечнокомпонентного уравнения поля Тоды, его $U-V$-пары, вспомогательных уравнений Лакса [11] и т. д.

В заключение с целью полноты изложения мы рассматриваем продолжение пуассоновых структур второго и третьего порядков, существующих для некоторых $R$-операторов на $\mathfrak{g}$, на классический дубль. Оказывается, что квадратичные и кубичные структуры всегда продолжаются на классический дубль $\mathcal{G}$, если они существуют на алгебре Ли g. Однако их применение в теории солитонов ограничивается тем фактом, что используемые факторпространства (пуассоновы пространства для линейной $\mathcal{R}$-скобки на $\mathcal{G}$ ) не являются, вообще говоря, пуассоновыми пространствами для квадратичной и кубичной скобок.

Структура настоящей работы следующая: в разделе 2 мы вводим основные определения и обозначения; в разделе 3 мы используем классический дубль для получения коммутативных семейств на $\mathfrak{g}^{*}$ и его расширениях; в разделе 4 мы применяем полученные результаты для конструирования уравнений нулевой кривиз- 
ны с $\mathfrak{g}$-значными $U-V$-парами и иллюстрируем этот подход примерами абелевого и неабелевого уравнений Тоды; наконец, в разделе 5 мы рассматриваем продолжение квадратичных и кубичных пуассоновых структур на классический дубль.

\section{2. ОПРЕДЕЛЕНИЯ И ОБОЗНАЧЕНИЯ}

2.1. Алгебры Ли и классические $R$-операторы. Пусть $\mathfrak{g}$ - алгебра Ли (конечно- или бесконечномерная) со скобкой Ли $[\cdot, \cdot]$, пусть $R: \mathfrak{g} \rightarrow \mathfrak{g}$ - линейный оператор. Оператор $R$ называется классическим $R$-оператором, если он удовлетворяет модифицированному уравнению Янга-Бакстера [10]:

$$
R([R(X), Y]+[X, R(Y)])-[R(X), R(Y)]=[X, Y]
$$

для любых $X, Y \in \mathfrak{g}$. Используя классический $R$-оператор, можно задать другую скобку Ли на $\mathfrak{g}$ следующей формулой [10]:

$$
[X, Y]_{R}=[R(X), Y]+[X, R(Y)], \quad X, Y \in \mathfrak{g} .
$$

Мы будем обозначать линейное пространство $\mathfrak{g}$, оснащенное скобкой $[\cdot, \cdot]_{R}$, через $\mathfrak{g}_{R}$. Мы будем использовать также следующие обозначения: $R_{ \pm} \equiv R \pm \mathrm{Id}$.

Известно [10], что образы $\mathfrak{g}_{R_{ \pm}}=\operatorname{Im} R_{ \pm}$отображений $R_{ \pm}$определяют подалгебры Ли $\mathfrak{g}_{R_{ \pm}} \subset \mathfrak{g}$. Как легко увидеть из определения, $\mathfrak{g}_{R_{+}}+\mathfrak{g}_{R_{-}}=\mathfrak{g}$, но, вообще говоря, эта сумма не является прямой суммой векторных пространств, т. е. в общем случае $\mathfrak{g}_{R_{+}} \cap \mathfrak{g}_{R_{-}} \neq 0$.

ЗАмЕчАниЕ 1. Ситуация намного упрощается в случае, когда алгебра Ли $\mathfrak{g}$ оснащена так называемым разложением AKC в прямую сумму двух подалгебр Ли: $\mathfrak{g}=\mathfrak{g}_{+}+\mathfrak{g}_{-}$. Действительно, если $P_{ \pm}$являются операторами, проецирующими на подалгебры $\mathfrak{g}_{ \pm}$, то $R=P_{+}-P_{-}$является классической $R$-матрицей [10]. Легко видеть, что в этом случае операторы $R_{ \pm}$пропорциональны операторам проецирования на подалгебры $\mathfrak{g}_{ \pm}$соответственно, $R_{ \pm}= \pm 2 P_{ \pm}$. Из этого следует также, что $\mathfrak{g}_{R_{ \pm}} \equiv \mathfrak{g}_{ \pm}$и $\mathfrak{g}_{R_{+}} \cap \mathfrak{g}_{R_{-}}=0$. Более того, известно [10], что в этом случае имеет место разложение $\mathfrak{g}_{R}=\mathfrak{g}_{+} \ominus \mathfrak{g}_{-}$.

2.2. Классический дубль. Рассмотрим “дубль” алгебры Ли g, т. е. прямую сумму $\mathcal{G}=\mathfrak{g} \oplus \mathfrak{g}$. Отождествим элемент $\mathcal{X} \in \mathcal{G}$ с вектор-столбцом $\mathcal{X}=\left(\begin{array}{l}X_{1} \\ X_{2}\end{array}\right)$, где $X_{i} \in \mathfrak{g}$. Скобка Ли двух элементов $\mathcal{X}, \mathcal{Y} \in \mathcal{G}$ задается стандартной формулой

$$
[\mathcal{X}, \mathcal{Y}]=\left(\begin{array}{l}
{\left[X_{1}, Y_{1}\right]} \\
{\left[X_{2}, Y_{2}\right]}
\end{array}\right)
$$

Следующая теорема была доказана в монографии [12].

Теорема 2.1. 1. Для произволъного классического $R$-оператора на $\mathfrak{g}$ линейный оператор на дубле, определяемый формулой

$$
\mathcal{R}=\left(\begin{array}{cc}
R & -R_{-} \\
R_{+} & -R
\end{array}\right),
$$

является классическим $R$-оператором на $\mathcal{G}$. 
2. Соответствующая $R$-скобка $[\cdot, \cdot]_{\mathcal{R}}$ на $\mathcal{G}$ имеет вид

$$
[\mathcal{X}, \mathcal{Y}]_{\mathcal{R}}=\left(\begin{array}{c}
{\left[X_{1}, Y_{1}\right]_{R}-\left(\left[X_{1}, R_{-}\left(Y_{2}\right)\right]+\left[R_{-}\left(X_{2}\right), Y_{1}\right]\right)} \\
-\left[X_{2}, Y_{2}\right]_{R}+\left(\left[X_{2}, R_{+}\left(Y_{1}\right)\right]+\left[R_{+}\left(X_{1}\right), Y_{2}\right]\right)
\end{array}\right) .
$$

3. R-onepamop $\mathcal{R}$ uмeem mun $A K C$.

Для удобства читателя мы приведем краткое доказательство п. 3 теоремы. Для этого рассмотрим следующие операторы:

$$
\mathcal{R}_{+}=\mathcal{R}+\mathrm{Id}=\left(\begin{array}{ll}
R_{+} & -R_{-} \\
R_{+} & -R_{-}
\end{array}\right), \quad \mathcal{R}_{-}=\mathcal{R}-\mathrm{Id}=\left(\begin{array}{ll}
R_{-} & -R_{-} \\
R_{+} & -R_{+}
\end{array}\right) .
$$

Введем обозначения $\mathcal{G}_{\mathcal{R}_{ \pm}}=\operatorname{Im} R_{ \pm}$для соответствующих подалгебр Ли. Легко видеть, что

$$
\mathcal{R}_{+}(\mathcal{X})=\left(\begin{array}{l}
R_{+}\left(X_{1}\right)-R_{-}\left(X_{2}\right) \\
R_{+}\left(X_{1}\right)-R_{-}\left(X_{2}\right)
\end{array}\right), \quad \mathcal{R}_{-}(\mathcal{X})=\left(\begin{array}{l}
R_{-}\left(X_{1}-X_{2}\right) \\
R_{+}\left(X_{1}-X_{2}\right)
\end{array}\right) .
$$

Из этого следует, что $\mathcal{G}_{\mathcal{R}_{+}} \equiv \mathcal{G}_{\mathrm{d}} \simeq \mathfrak{g}, \mathcal{G}_{\mathcal{R}_{-}} \simeq \mathfrak{g}_{R}$, где

$$
\mathcal{G}_{\mathrm{d}}=\left\{\left(\begin{array}{l}
X \\
X
\end{array}\right) \mid X \in \mathfrak{g}\right\}, \quad \mathcal{G}_{\mathcal{R}_{-}}=\left\{\left(\begin{array}{l}
R_{-}(X) \\
R_{+}(X)
\end{array}\right) \mid X \in \mathfrak{g}\right\} .
$$

Нетрудно заметить, что $\operatorname{Ker} \mathcal{R}_{+}=\operatorname{Im} \mathcal{R}_{-}$и $\operatorname{Ker} \mathcal{R}_{-}=\operatorname{Im} \mathcal{R}_{+}$. Следовательно, разложение $\mathcal{G}=\mathcal{G}_{\mathcal{R}_{+}}+\mathcal{G}_{\mathcal{R}_{-}}$является разложением в прямую сумму векторных пространств и оператор $\mathcal{R}$ имеет тип $\mathrm{AKC}$, т. е. $\mathcal{R}=\mathcal{P}_{\mathcal{G}_{\mathcal{R}_{+}}}-\mathcal{P}_{\mathcal{G}_{R_{-}}}$. Этот факт также следует из легко доказываемых тождеств $\mathcal{R}_{ \pm}^{2}=2 \mathcal{R}_{ \pm}, \mathcal{R}_{+} \mathcal{R}_{-}=0$, подразумевающих, что операторы $\mathcal{R}_{ \pm}$пропорциональны проекционным операторам и их образы не пересекаются. Отсюда мы получаем $\mathcal{G}_{\mathcal{R}}=\mathcal{G}_{\mathcal{R}_{+}} \ominus \mathcal{G}_{R_{-}}$. Для $\mathcal{R}$-скобки на дубле это означает следующее: $[\mathcal{X}, \mathcal{Y}]_{\mathcal{R}}=2\left(\left[\mathcal{X}_{+}, \mathcal{Y}_{+}\right]-\left[\mathcal{X}_{-}, \mathcal{Y}_{-}\right]\right)$, где $\mathcal{X}_{ \pm} \equiv \mathcal{R}_{ \pm}(\mathcal{X})$ или, в более явной форме,

$$
\begin{aligned}
{[\mathcal{X}, \mathcal{Y}]_{\mathcal{R}}=\left(\begin{array}{l}
{\left[\left(R_{+}\left(X_{1}\right)-R_{-}\left(X_{2}\right)\right),\left(R_{+}\left(Y_{1}\right)-R_{-}\left(Y_{2}\right)\right)\right]} \\
{\left[\left(R_{+}\left(X_{1}\right)-R_{-}\left(X_{2}\right)\right),\left(R_{+}\left(Y_{1}\right)-R_{-}\left(Y_{2}\right)\right)\right]}
\end{array}\right)-} \\
\\
\quad-\left(\begin{array}{l}
{\left[R_{-}\left(X_{1}-X_{2}\right), R_{-}\left(Y_{1}-Y_{2}\right)\right]} \\
{\left[R_{+}\left(X_{1}-X_{2}\right), R_{+}\left(Y_{1}-Y_{2}\right)\right]}
\end{array}\right) .
\end{aligned}
$$

ЗАмЕчание 2 . В случае $R$-операторов $\mathrm{AKC} \mathrm{все} \mathrm{формулы} \mathrm{этого} \mathrm{раздела} \mathrm{суще-}$ ственно упрощаются. В частности, действие $R$-оператора $\mathcal{R}$ на элемент $\mathcal{X}$ задается формулой

$$
\mathcal{R}(\mathcal{X})=\left(\begin{array}{l}
X_{1}^{+}-X_{1}^{-}+2 X_{2}^{-} \\
2 X_{1}^{+}-X_{2}^{+}+X_{2}^{-}
\end{array}\right)
$$

a $R$-скобка (1) записывается следующим образом:

$$
[\mathcal{X}, \mathcal{Y}]_{\mathcal{R}}=\left(\begin{array}{c}
{\left[X_{1}^{+}, Y_{1}^{+}\right]-\left[X_{1}^{-}, Y_{1}^{-}\right]-\left(\left[X_{1}, Y_{2}^{-}\right]+\left[X_{2}^{-}, Y_{1}\right]\right)} \\
-\left[X_{2}^{+}, Y_{2}^{+}\right]+\left[X_{2}^{-}, Y_{2}^{-}\right]+\left(\left[X_{2}, Y_{1}^{+}\right]+\left[X_{1}^{+}, Y_{2}\right]\right)
\end{array}\right),
$$

где $X_{i}=X_{i}^{+}+X_{i}^{-}, Y_{i}=Y_{i}^{+}+Y_{i}^{-}$и $X_{i}^{ \pm}=P_{ \pm}(X), Y_{i}^{ \pm}=P_{ \pm}(Y), i=1,2$. 
2.3. Дуальные пространства скобки Ли-Пуассона и инвариантные функции. Пусть $\mathfrak{g}^{*}-$ пространство, дуальное к алгебре Ли $\mathfrak{g}$, и $\langle\cdot, \cdot\rangle: \mathfrak{g}^{*} \times \mathfrak{g} \rightarrow \mathbb{C}-$ естественное спаривание между $\mathfrak{g}^{*}$ и $\mathfrak{g}$. Пусть $\left\{X_{i} \mid i \in I\right\}-$ базис алгебры Ли $\mathfrak{g}$, где множество индексов $I$ конечно в случае конечномерных алгебр Ли или счетно в бесконечномерном случае. Пусть $\left\{X_{i}^{*} \mid i \in I\right\}$ - базис в дуальном пространстве $\mathfrak{g}^{*}$, $\left\langle X_{j}^{*}, X_{i}\right\rangle=\delta_{i j}$. Пусть $L=\sum_{i \in I} L_{i} X_{i}^{*} \in \mathfrak{g}^{*}$ - элемент общего положения в пространстве $\mathfrak{g}^{*}, L_{i}$ - координатные функции на $\mathfrak{g}^{*}$. Рассмотрим стандартную скобку Ли-Пуассона между функциями $F_{1}, F_{2} \in C^{\infty}\left(\mathfrak{g}^{*}\right)$ на пространстве $\mathfrak{g}^{*}$ :

$$
\left\{F_{1}(L), F_{2}(L)\right\}=\left\langle L,\left[\nabla F_{1}, \nabla F_{2}\right]\right\rangle, \quad \nabla F_{k}(L)=\sum_{i \in I} \frac{\partial F_{k}(L)}{\partial L_{i}} X_{i}
$$

здесь $\nabla F_{k}(L)$ - так называемый алгеброзначный градиент функции $F_{k}, k=1,2$. При этом $R$-оператор задает $R$-скобку на $\mathfrak{g}^{*}$ следующим образом [10]:

$$
\left\{F_{1}(L), F_{2}(L)\right\}_{R}=\left\langle L,\left[\nabla F_{1}, \nabla F_{2}\right]_{R}\right\rangle .
$$

Рассмотрим пространство $\mathcal{G}^{*}$, дуальное к классическому дублю. Определим его элементы $\mathcal{L} \in \mathfrak{g}^{*} \oplus \mathfrak{g}^{*}$ как вектор-столбцы $\mathcal{L}=\left(\begin{array}{c}L_{1} \\ L_{2}\end{array}\right)$, где $L_{1}, L_{2} \in \mathfrak{g}^{*}$, а спаривание между $\mathcal{L} \in \mathcal{G}^{*}$ и $\mathcal{X} \in \mathcal{G}$ зададим следующим образом:

$$
\langle\mathcal{L}, \mathcal{X}\rangle=\left\langle L_{1}, X_{1}\right\rangle+\left\langle L_{2}, X_{2}\right\rangle
$$

Оно также определяет стандартную скобку Ли-Пуассона на $\mathcal{G}^{*}$,

$$
\left\{F_{1}(\mathcal{L}), F_{2}(\mathcal{L})\right\}=\left\langle\mathcal{L},\left[\widetilde{\nabla} F_{1}, \widetilde{\nabla} F_{2}\right]\right\rangle
$$

и $R$-скобку на $\mathcal{G}^{*}$, соответствующую $R$-оператору $\mathcal{R}$,

$$
\left\{F_{1}(\mathcal{L}), F_{2}(\mathcal{L})\right\}_{\mathcal{R}}=\left\langle\mathcal{L},\left[\widetilde{\nabla} F_{1}, \widetilde{\nabla} F_{2}\right]_{\mathcal{R}}\right\rangle, \quad \widetilde{\nabla} F=\left(\begin{array}{c}
\nabla_{1} F \\
\nabla_{2} F
\end{array}\right)
$$

здесь $\nabla_{1,2} F$ - алгеброзначные градиенты функции $F$ по переменным $L_{1,2}$.

Обозначим через $R^{*}$ сопряженный к $R$ оператор:

$$
R^{*}: \mathfrak{g}^{*} \rightarrow \mathfrak{g}^{*}, \quad\left\langle R^{*}(L), X\right\rangle \equiv\langle L, R(X)\rangle .
$$

Легко видеть, что операторы, сопряженные к операторам $\mathcal{R}_{ \pm}$, имеют следующий вид:

$$
\mathcal{R}_{+}^{*}(\mathcal{L})=\left(\begin{array}{c}
R_{+}^{*}\left(L_{1}\right)+R_{+}^{*}\left(L_{2}\right) \\
-\left(R_{-}^{*}\left(L_{1}\right)+R_{-}^{*}\left(L_{2}\right)\right)
\end{array}\right), \quad \mathcal{R}_{-}^{*}(\mathcal{L})=\left(\begin{array}{c}
R_{-}^{*}\left(L_{1}\right)+R_{+}^{*}\left(L_{2}\right) \\
-\left(R_{-}^{*}\left(L_{1}\right)+R_{+}^{*}\left(L_{2}\right)\right)
\end{array}\right) .
$$

Мы будем использовать эти явные формулы при конструировании пуассон-коммутирующих функций.

Далее нам также потребуется явная форма функций Казимира алгебры $\mathcal{G}$. Пусть $I(L) \in I^{G}\left(\mathfrak{g}^{*}\right)$ - функция Казимира алгебры $\mathfrak{g}$, т. е. $\{I(L), F(L)\}=0$ для всех $F(L) \in S\left(\mathfrak{g}^{*}\right)$. Обозначим через $\left\{I_{k}(L)\right\}_{k \in K}$ набор генераторов кольца функции Казимира на $\mathfrak{g}^{*}$. Здесь набор индексов $K$ может быть бесконечным, если алгебра Ли бесконечномерна. 
ЛЕмма 2.1. Колъцо функции Казимира алгебры $\mathcal{G}$ порождается следующими функциями:

$$
I_{k, 1}(\mathcal{L}) \equiv I_{k}\left(L_{1}\right), \quad I_{k, 2}(\mathcal{L}) \equiv I_{k}\left(L_{2}\right), \quad k \in K
$$

ДоКАЗАТЕЛЬСТВО этой леммы очевидно.

Дадим краткие комментарии по поводу квадратичных функций Казимира и коммутирующих функций, которые могут быть получены с их помощью. Пусть (·, · ) билинейная инвариантная форма на $\mathfrak{g}$. C ее помощью можно отождествить пространства $\mathfrak{g}$ и $\mathfrak{g}^{*}$. В этом случае мы имеем следующие очевидные квадратичные функции Казимира (или генерирующие функции формальных функций Казимира в случае алгебр петель):

$$
I_{2,1}=\frac{1}{2}\left(L_{1}, L_{1}\right), \quad I_{2,2}=\frac{1}{2}\left(L_{2}, L_{2}\right) .
$$

\section{3. КЛАССИЧЕСКИЙ ДУБЛЬ И КОММУТИРУЮЩИЕ ПОТОКИ}

Чтобы перейти к основной конструкции, рассматривающейся в настоящей статье, необходимо сформулировать следующую теорему, которая является следствием общей теории $R$-скобок [10], примененной к классическому дублю.

ТеОРема 3.1. 1. Функции Казимира $I_{k, \epsilon}(\mathcal{L}), \epsilon=1,2$, алгебры $\mathcal{G}$ коммутируют по отношению $к$ скобкам Пуассона $\{\cdot, \cdot\}_{\mathcal{R}}$ на $\mathcal{G}^{*}$.

2. Гамильтоновы потоки

$$
\frac{d}{d t_{k}^{\epsilon}} F(\mathcal{L})=\left\{F(\mathcal{L}), I_{k, \epsilon}\right\}_{\mathcal{R}}, \quad k \in K, \quad \epsilon=1,2,
$$

порожденные функииями $I_{k, \epsilon}$, записываются в форме Эйлера-Арнолъда:

$$
\frac{d \mathcal{L}}{d t_{k}^{\epsilon}}=\operatorname{ad}_{\mathcal{R}_{+} \tilde{\nabla} I_{k, \epsilon}}^{*} \mathcal{L} .
$$

Рассмотрим кратко коммутирующие потоки на алгебре $\mathfrak{g}$ и ее расширениях, которые могут быть получены с использованием теории классического дубля. С этой целью напомним, что проекция на фактор-алгебру является каноническим гомоморфизмом, что позволяет получить следующее следствие.

СлеДСТвИЕ 3.1. Пусть $J-$ идеал в алгебре $\mathcal{G}_{\mathcal{R}}$. Обозначим через $\pi: \mathcal{G} \rightarrow \mathcal{G} / J$ проекцию на фактор-алгебру. Пусть $\pi^{*}$ - дуальное отображение, тогда:

1) бункиии $I_{k, \epsilon}\left(\pi^{*}(\mathcal{L})\right)$ коммутируют относителъно скобок Пуассона $\{\cdot, \cdot\}_{\mathcal{R}}$ на $(\mathcal{G} / J)^{*}$

2 ) гамильтоновы потоки функции $I_{k, \epsilon}\left(\pi^{*}(\mathcal{L})\right)$ записъваются в форме Эйлера-Арнольда

$$
\pi^{*}\left(\frac{d \mathcal{L}}{d t_{k}^{\epsilon}}\right)=\operatorname{ad}_{M_{k, \epsilon}}^{*} \pi^{*}(\mathcal{L}),
$$

где

$$
M_{k, \epsilon}=\mathcal{R}_{+}\left(\pi \widetilde{\nabla} I_{k, \epsilon}\left(\pi^{*}(\mathcal{L})\right)\right), \quad k \in K, \quad \epsilon=1,2 .
$$


Предположим, что существуют нетривиальные идеалы $J_{R_{ \pm}} \subset \mathfrak{g}_{R_{ \pm}}$такие, что фактор-алгебры $\mathfrak{g}_{R_{ \pm}} / J_{R_{ \pm}}$конечномерны. В таком случае, применив следствие 3.1 и взяв фактор по идеалу $J=J_{R_{+}}+J_{R_{-}}$, мы получим пуассон-коммутирующий набор функций на пространстве, дуальном к расширению алгебры $\mathfrak{g}$ конечномерными алгебрами. Действительно, $\mathcal{G}_{\mathcal{R}} / J=\left(\mathcal{G}_{\mathcal{R}_{+}} \ominus \mathcal{G}_{\mathcal{R}_{-}}\right) / J \simeq \mathfrak{g} \ominus \mathfrak{a}$, где $\mathfrak{a} \simeq \mathcal{G}_{\mathcal{R}_{-}} / J$.

ЗАмечАниЕ 3. Для фактор-алгебр, описаных выше, $M$-операторы из уравнений Лакса (8) имеют вид

$$
M_{k, 1}=\left(\begin{array}{c}
R_{+}\left(\nabla I_{k, 1}\left(\pi^{*}(\mathcal{L})\right)\right) \\
R_{+}\left(\nabla I_{k, 1}\left(\pi^{*}(\mathcal{L})\right)\right)
\end{array}\right), \quad M_{k, 2}=-\left(\begin{array}{c}
R_{-}\left(\nabla I_{k, 2}\left(\pi^{*}(\mathcal{L})\right)\right) \\
R_{-}\left(\nabla I_{k, 2}\left(\pi^{*}(\mathcal{L})\right)\right)
\end{array}\right),
$$

т. е. принадлежат диагональной подалгебре и могут быть отождествлены с элементами алгебры g. Этот факт будет использован при выводе уравнений нулевой кривизны со значениями в алгебре Ли g. Отметим, что соответствующие уравнения Лакса (8), динамические переменные, скобки Пуассона и т. д. принадлежат дублю алгебры $\mathfrak{g}$. Отметим также, что отсутствие проекционного оператора $\pi$ в формулах для $M$-операторов объясняется тем, что выбор идеала обеспечивает равенство $\mathcal{R}_{+} \pi=\mathcal{R}_{+}$.

Пример 1. Рассмотрим $R$-операторы AKC: $R=P_{+}-P_{-}$. Опишем в явном виде фактор-алгебры $\mathcal{G}_{\mathcal{R}} / J$ и соответствующие дуальные пространства. В этом случае мы имеем $\mathfrak{g}=\mathfrak{g}_{+}+\mathfrak{g}_{-}, \mathfrak{g}_{R_{ \pm}}=\mathfrak{g}_{ \pm}$и $J_{R_{ \pm}} \equiv J_{ \pm}$- идеалы в $\mathfrak{g}_{ \pm}$. Элементы соответствующих фактор-алгебр $\mathcal{G}_{\mathcal{R}} / J$, где $J=J_{+}+J_{-}$, задаются как

$$
\mathcal{X}=\left(\begin{array}{c}
X_{1}^{+}+X_{1}^{-^{\prime}} \\
X_{2}^{-}+X_{2}^{+^{\prime}}
\end{array}\right), \quad X_{1}^{-^{\prime}} \in \mathfrak{g}_{-} / J_{-}, \quad X_{2}^{+^{\prime}} \in \mathfrak{g}_{+} / J_{+} .
$$

Соответствущее дуальное пространство состоит из элементов вида

$$
\mathcal{L}=\left(\begin{array}{c}
L_{1}^{+}+L_{1}^{-^{\prime}} \\
L_{2}^{-}+L_{2}^{+^{\prime}}
\end{array}\right)
$$

где $L_{1}^{-^{\prime}} \in\left(\mathfrak{g}_{-} / J_{-}\right)^{*}, L_{1}^{+} \in\left(\mathfrak{g}_{+}\right)^{*}, L_{2}^{-} \in\left(\mathfrak{g}_{-}\right)^{*},{L_{2}^{+}}^{\prime} \in\left(\mathfrak{g}_{+} / J_{+}\right)^{*}$.

В следующем пункте мы рассмотрим детальнее случаи $\mathfrak{a} \simeq \mathcal{G}_{\mathcal{R}_{-}} / J=0$ и абелевой алгебры $\mathfrak{a}$, которые приводят к коммутативной алгебре интегралов на само́м пространстве $\mathfrak{g}^{*}$.

3.1. Дуальная $R$-матричная коммутативность. Рассмотрим следствие из общей теоремы 3.1, которое потребуется нам для построения функций, пуассон-коммутирующих по отношению к стандартной скобке Ли-Пуассона $\{\cdot, \cdot\}$ на $\mathfrak{g}^{*}$. Имеет место следующая теорема.

ТЕОРема 3.2. 1. Функиии $I_{k}\left(R_{ \pm}^{*}(L)\right)$ на $\mathfrak{g}^{*}$ генерируют абелеву подалгебру в $C^{\infty}\left(\mathfrak{g}^{*}\right)$ по отношению $\kappa$ исходной скобке Ли-Пуассона $\{\cdot, \cdot\}$ на $\mathfrak{g}^{*}$ :

$$
\begin{aligned}
& \left\{I_{k}\left(R_{+}^{*}(L)\right), I_{l}\left(R_{+}^{*}(L)\right)\right\}=0, \\
& \left\{I_{k}\left(R_{-}^{*}(L)\right), I_{l}\left(R_{-}^{*}(L)\right)\right\}=0, \\
& \left\{I_{k}\left(R_{+}^{*}(L)\right), I_{l}\left(R_{-}^{*}(L)\right)\right\}=0 .
\end{aligned}
$$


2. Гамильтоновъ уравнения, отвечающие гамильтонианам $I_{k}^{R_{ \pm}}(L)$, могут быть записаны в форме Эйлера-Арнольда:

$$
\frac{d L}{d t_{k}^{ \pm}}=\operatorname{ad}_{M_{k}^{ \pm}}^{*} L, \quad M_{k}^{ \pm}=\nabla I_{k}\left(R_{ \pm}^{*}(L)\right) .
$$

ДоКАЗАТЕЛЬСтво. Возьмем проекции функций $I_{k, \epsilon}(\mathcal{L}), \epsilon=1,2$, на пространство, дуальное к фактор-алгебре $\mathcal{G}_{\mathcal{R}} / \mathcal{G}_{R_{-}}$, которое совпадает с пространством, дуальным к подалгебре $\mathcal{G}_{R_{+}}$. Используя формулы $(5),(6)$, мы получаем следующее выражение для проекций функций Казимира:

$$
I_{k, 1}\left(P_{\mathcal{G}_{R_{+}}}^{*}(\mathcal{L})\right)=I_{k}\left(R_{+}^{*}\left(L_{1}+L_{2}\right)\right), \quad I_{k, 2}\left(P_{\mathcal{G}_{R_{+}}}^{*}(\mathcal{L})\right)=I_{k}\left(R_{-}^{*}\left(L_{1}+L_{2}\right)\right),
$$

где мы приняли во внимание, что $P_{\mathcal{G}_{R_{+}}^{*}}^{*}=\mathcal{R}_{+}^{*} / 2$ и предположили, что $I_{k}-$ однородная функция от $L$.

Заметим, что, поскольку $\mathcal{R}_{+}-$проекционный оператор и соответствующий $R$-оператор $\mathcal{R}$ имеет тип $\mathrm{AKC}$, легко вывести следующее равенство:

$$
\left\{F\left(\mathcal{R}_{+}^{*}(\mathcal{L})\right), G\left(\mathcal{R}_{+}^{*}(\mathcal{L})\right)\right\}=\left\{F\left(\mathcal{R}_{+}^{*}(\mathcal{L})\right), G\left(\mathcal{R}_{+}^{*}(\mathcal{L})\right)\right\}_{\mathcal{R}} .
$$

Используя тот факт, что проекция на фактор-алгебру является каноническим гомоморфизмом, мы получаем соотношение

$$
\left\{F\left(\mathcal{R}_{ \pm}^{*}(\mathcal{L})\right), G\left(\mathcal{R}_{ \pm}^{*}(\mathcal{L})\right)\right\}_{\mathcal{R}}=\left.\left(\{F(\mathcal{L}), G(\mathcal{L})\}_{\mathcal{R}}\right)\right|_{\mathcal{L}=\mathcal{R}_{ \pm}^{*}(\mathcal{L})}
$$

Отсюда, положив $F=I_{k, \epsilon}, G=I_{l, \epsilon^{\prime}}$, мы получим

$$
\left\{I_{k, \epsilon}\left(\mathcal{R}_{+}^{*}(\mathcal{L})\right), I_{l, \epsilon^{\prime}}\left(\mathcal{R}_{+}^{*}(\mathcal{L})\right)\right\}=\left.\left(\left\{I_{k, \epsilon}(\mathcal{L}), I_{l, \epsilon^{\prime}}(\mathcal{L})\right\}_{\mathcal{R}}\right)\right|_{\mathcal{L}=\mathcal{R}_{+}^{*}(\mathcal{L})}, \quad \epsilon, \epsilon^{\prime}=1,2 .
$$

С другой стороны, $\left\{I_{k, \epsilon}(\mathcal{L}), I_{l, \epsilon^{\prime}}(\mathcal{L})\right\}_{\mathcal{R}}=0$ в силу теоремы 3.1.

Используя явную форму функций $I_{k, \epsilon}(\mathcal{L})$, мы, наконец, получаем следующие равенства:

$$
\begin{aligned}
& \left\{I_{k}\left(R_{+}^{*}\left(L_{1}+L_{2}\right)\right), I_{l}\left(R_{+}^{*}\left(L_{1}+L_{2}\right)\right)\right\}=0, \\
& \left\{I_{k}\left(R_{-}^{*}\left(L_{1}+L_{2}\right)\right), I_{l}\left(R_{-}^{*}\left(L_{1}+L_{2}\right)\right)\right\}=0, \\
& \left\{I_{k}\left(R_{+}^{*}\left(L_{1}+L_{2}\right)\right), I_{l}\left(R_{-}^{*}\left(L_{1}+L_{2}\right)\right)\right\}=0 .
\end{aligned}
$$

Теперь для завершения доказательства п. 1 теоремы остается заметить, что элементы вида $L \equiv L_{1}+L_{2}$ принадлежат пространству $\left(\mathcal{G}_{\mathrm{d}}\right)^{*}$ и соответствующие координатные функции образуют алгебру Ли, изоморфную $(\mathfrak{g},\{\cdot, \cdot\})$, по отношению к исходной скобке Ли-Пуассона на $\mathcal{G}$.

Второе утверждение теоремы доказывается на основе п. 2 теоремы 3.1. Оно может быть также доказано с использованием того факта, что любое гамильтоново уравнение на $\mathfrak{g}^{*}$ переписывается в виде уравнения Эйлера-Арнольда. Теорема доказана.

ЗАмЕчАниЕ 4. В работе [8] данная теорема была доказана без использования классического дубля. Однако доказательство, основанное на дубле, является более простым и делает теорему 3.2 понятной с точки зрения стандартной $R$-операторной схемы. 
Пример 2. Рассмотрим детальнее случай $R=P_{+}-P_{-}$и приведем явный вид алгебры Ли $\mathcal{G}_{\mathcal{R}_{+}}$, реализованной как фактор-алгебра. Мы имеем

$$
\operatorname{Ker} \mathcal{R}_{+}=\operatorname{Im} \mathcal{R}_{-}=\left(\begin{array}{c}
X_{1}^{-} \\
X_{2}^{+}
\end{array}\right)
$$

Соответствущая фактор-алгебра $\mathcal{G}_{\mathcal{R}} / \operatorname{Im} \mathcal{R}_{-}$может быть отождествлена с линейным пространством, состоящим из элементов вида $\mathcal{X}=\left(\begin{array}{c}X_{1}^{+} \\ X_{2}^{-}\end{array}\right)$. Это пространство изоморфно алгебре $\mathcal{G}_{\mathcal{R}_{+}}$, и изоморфизм задается отображением $\mathcal{R}_{+}$:

$$
\left(\begin{array}{l}
X_{1}^{+} \\
X_{2}^{-}
\end{array}\right) \rightarrow\left(\begin{array}{l}
X_{1}^{+}-X_{2}^{-} \\
X_{1}^{+}-X_{2}^{-}
\end{array}\right)
$$

Соответствующее дуальное пространство состоит из элементов $\mathcal{L}=\left(\begin{array}{c}L_{1}^{+} \\ L_{2}^{-}\end{array}\right)$. Заметим, что такие элементы могут быть отождествлены с элементами $L=L_{1}^{+}+L_{2}^{-}$ линейного пространства $\mathfrak{g}^{*}$. Более того, соответствующая скобка Ли-Пуассона этих элементов, вычисленная на фактор-алгебре $\mathcal{G}_{\mathcal{R}} / \operatorname{Im} \mathcal{R}_{-}$, совпадает со стандартной скобкой Ли-Пуассона элемента $L=L^{+}+L^{-}$на $\mathfrak{g}^{*}$.

Функции Казимира $I_{k, 1}(\mathcal{L})$ и $I_{k, 2}(\mathcal{L})$, ограниченные на пространство, дуальное к фактор-алгебре, совпадают с функциями $I_{k}\left(L_{1}^{+}\right)$и $I_{k}\left(L_{2}^{-}\right)$соответственно. После описанного выше отождествления они переходят в функции $I_{k}\left(L^{+}\right)$и $I_{k}\left(L^{-}\right)$на $\mathfrak{g}^{*}$.

3.2. Сдвиг аргумента и коммутативные подалгебры. Рассмотрим коммутативные подалгебры функций на $\mathfrak{g}^{*}$, которые обобщают коммутативные алгебры, сконструированные в предыдущем пункте. Эти подалгебры зависят от дополнительных параметров, получаемых с использованием теории классического дубля. Метод, который позволяет ввести указанные дополнительные параметры в коммутативные подалгебры, является обобщением так называемого метода сдвига аргумента. Справедлива следующая теорема.

Теорема 3.3. Пусть $c_{ \pm}-$постоянные элементы пространств $\mathfrak{g}_{R_{ \pm}}^{*}$ такие, что

$$
c_{ \pm} \perp\left(\left[\mathfrak{g}_{R_{+}}, \mathfrak{g}_{R_{+}}\right] \cup\left[\mathfrak{g}_{R_{-}}, \mathfrak{g}_{R_{-}}\right]\right),
$$

и пусть $I_{k}(L), I_{l}(L)$ - функции Казимира алгебры $\mathfrak{g}$. Тогда имеют место следующие утверждения.

1. Справедливы равенства

$$
\begin{aligned}
& \left\{I_{k}\left(R_{+}^{*}(L)+c_{-}\right), I_{l}\left(R_{+}^{*}(L)+c_{-}\right)\right\}_{c}=0, \\
& \left\{I_{k}\left(R_{-}^{*}(L)+c_{+}\right), I_{l}\left(R_{-}^{*}(L)+c_{+}\right)\right\}_{c}=0, \\
& \left\{I_{k}\left(R_{+}^{*}(L)+c_{-}\right), I_{l}\left(R_{-}^{*}(L)+c_{+}\right)\right\}_{c}=0,
\end{aligned}
$$

где $\{\cdot, \cdot\}_{c}-$ "сдвинутая" скобка Пуассона:

$$
\left\{F_{1}(L), F_{2}(L)\right\}_{c}=\left\langle L,\left[\nabla F_{1}, \nabla F_{2}\right]\right\rangle+\left\langle c_{-}-c_{+},\left[\nabla F_{1}, \nabla F_{2}\right]\right\rangle .
$$

2. Соответствующие гамильтоновы уравнения записываются в форме уравнений Эйлера-Арнольда:

$$
\frac{d L}{d t_{k}^{ \pm}}=\operatorname{ad}_{\nabla I_{k}\left(R_{ \pm}^{*}(L)+c_{\mp}\right)}^{*}\left(L+c_{-}-c_{+}\right) .
$$


ДокАЗАТЕЛьство. Для доказательства п. 1 теоремы примем во внимание, что $\mathcal{G}_{\mathcal{R}}=\mathcal{G}_{\mathcal{R}_{+}} \ominus \mathcal{G}_{\mathcal{R}_{-}}$. Следовательно, $\left[\mathcal{G}_{\mathcal{R}}, \mathcal{G}_{\mathcal{R}}\right]=\left[\mathcal{G}_{\mathcal{R}_{+}}, \mathcal{G}_{\mathcal{R}_{+}}\right] \ominus\left[\mathcal{G}_{\mathcal{R}_{-}}, \mathcal{G}_{\mathcal{R}_{-}}\right]$. Опишем явно идеал $\left[\mathcal{G}_{\mathcal{R}_{-}}, \mathcal{G}_{\mathcal{R}_{-}}\right]$. Мы имеем

$$
\mathcal{G}_{\mathcal{R}_{-}}=\left\{\left(\begin{array}{c}
X_{1} \\
X_{2}
\end{array}\right) \mid X_{1} \in \mathfrak{g}_{R_{-}}, X_{2} \in \mathfrak{g}_{R_{+}}\right\} .
$$

Из этого следует, что элемент $C=\left(c_{1}, c_{2}\right) \in \mathcal{G}^{*}$ ортогонален $\left[\mathcal{G}_{\mathcal{R}_{-}}, \mathcal{G}_{\mathcal{R}_{-}}\right]$, если $c_{1} \perp\left[\mathfrak{g}_{R_{-}}, \mathfrak{g}_{R_{-}}\right]$и $c_{2} \perp\left[\mathfrak{g}_{R_{+}}, \mathfrak{g}_{R_{+}}\right]$. С другой стороны, из явной формы элементов $\mathcal{G}_{\mathcal{R}_{-}}^{*}$ следует, что $c_{2}=-c_{1}=-c$, и $C=(c,-c)$ является элементом пространства, дуального к подалгебре Ли $\mathcal{G}_{\mathcal{R}_{-}}$. Этот элемент ортогонален $\left[\mathcal{G}_{\mathcal{R}_{-}}, \mathcal{G}_{\mathcal{R}_{-}}\right]$, если $c \perp\left(\left[\mathfrak{g}_{R_{+}}, \mathfrak{g}_{R_{+}}\right] \cup\left[\mathfrak{g}_{R_{-}}, \mathfrak{g}_{R_{-}}\right]\right)$. Таким образом, факторизируя алгебру $\mathcal{G}_{\mathcal{R}}$ по идеалу $\left[\mathcal{G}_{\mathcal{R}_{-}}, \mathcal{G}_{\mathcal{R}_{-}}\right]$, принимая во внимание, что проекция на фактор-алгебру является каноническим гомоморфизмом, и применяя теорему $3.1 \mathrm{k}$ функциям Казимира $I_{k, \epsilon}, I_{l, \epsilon^{\prime}}$, мы получаем

$$
\begin{aligned}
& \left\{I_{k}\left(R_{+}^{*}\left(L_{1}+L_{2}\right)+c\right), I_{l}\left(R_{+}^{*}\left(L_{1}+L_{2}\right)+c\right)\right\}=0, \\
& \left\{I_{k}\left(R_{-}^{*}\left(L_{1}+L_{2}\right)+c\right), I_{l}\left(R_{-}^{*}\left(L_{1}+L_{2}\right)+c\right)\right\}=0, \\
& \left\{I_{k}\left(R_{+}^{*}\left(L_{1}+L_{2}\right)+c\right), I_{l}\left(R_{-}^{*}\left(L_{1}+L_{2}\right)+c\right)\right\}=0 .
\end{aligned}
$$

В результате мы получаем коммутативную подалгебру с элементом сдвига $c$, входящим симметрично как в “положительные", так и в "отрицательные" интегралы, т. е. элемент $c$ имеет компоненты, принадлежащие и $\mathfrak{g}_{R_{-}}^{*}$, и $\mathfrak{g}_{R_{+}}^{*}$. Заметим, что сдвиг принадлежащих $\mathfrak{g}_{R_{ \pm}}^{*}$ матриц Лакса на постоянный элемент из того же пространства $\mathfrak{g}_{R_{ \pm}}^{*}$ может быть удален путем замены переменных. Однако это ведет к изменению скобок Пуассона. Сделав такую замену и положив $c_{ \pm}= \pm R_{ \pm}^{*}(c), L=L_{1}+L_{2}$, мы получаем утверждение п. 1 теоремы.

Утверждение п. 2 доказывается аналогично утверждению п. 2 предыдущей теоремы. Теорема доказана.

ПримеР 3. Рассмотрим, как и в предыдущих примерах, $R$-операторы AKC: $R=$ $P_{+}-P_{-}$. Опишем в явном виде фактор-алгебру $\mathcal{G}_{\mathcal{R}} /\left[\mathcal{G}_{\mathcal{R}_{-}}, \mathcal{G}_{\mathcal{R}_{-}}\right]$и соответствующее дуальное пространство. Мы имеем $\left[\mathcal{G}_{\mathcal{R}_{-}}, \mathcal{G}_{\mathcal{R}_{-}}\right]=\left[\mathfrak{g}_{+}, \mathfrak{g}_{+}\right] \ominus\left[\mathfrak{g}_{-}, \mathfrak{g}_{-}\right]$. Элементы факторпространств $\mathcal{G}_{\mathcal{R}} /\left[\mathcal{G}_{\mathcal{R}_{-}}, \mathcal{G}_{\mathcal{R}_{-}}\right]$записываются как

$$
\mathcal{X}=\left(\begin{array}{l}
X_{1}^{+}+X_{1}^{-^{\prime}} \\
X_{2}^{-}+X_{2}^{+^{\prime}}
\end{array}\right)
$$

где $X_{1}^{+} \in \mathfrak{g}_{+}, X_{1}^{-^{\prime}} \in \mathfrak{g}_{-} /\left[\mathfrak{g}_{-}, \mathfrak{g}_{-}\right], X_{2}^{-} \in \mathfrak{g}_{-}, X_{2}^{+^{\prime}} \in \mathfrak{g}_{+} /\left[\mathfrak{g}_{+}, \mathfrak{g}_{+}\right]$. Соответствущее дуальное пространство состоит из элементов вида

$$
\left(\begin{array}{c}
L_{1}^{+}+L_{1}^{-^{\prime}} \\
L_{2}^{-}+L_{2}^{+^{\prime}}
\end{array}\right), \quad L_{1}^{-^{\prime}} \in\left(\mathfrak{g}_{-} /\left[\mathfrak{g}_{-}, \mathfrak{g}_{-}\right]\right)^{*}, \quad L_{2}^{+^{\prime}} \in\left(\mathfrak{g}_{+} /\left[\mathfrak{g}_{+}, \mathfrak{g}_{+}\right]\right)^{*} .
$$

Элементы $L_{1}^{-^{\prime}}, L_{2}^{+^{\prime}}$ постоянны по отношению к скобкам Пуассона на $\mathcal{G}_{\mathcal{R}} /\left[\mathcal{G}_{\mathcal{R}_{-}}, \mathcal{G}_{\mathcal{R}_{-}}\right]$, и можно положить $c_{-}=L_{1}^{-^{\prime}}$ и $c_{+}=L_{2}^{+^{\prime}}$.

Функции Казимира, ограниченные на дуальное пространство фактор-алгебры, это функции $I_{k}\left(L_{1}^{+}+{L_{1}^{-}}^{\prime}\right)$ и $I_{l}\left(L_{2}^{-}+{L_{2}^{+}}^{\prime}\right)$. Используя те же аргументы, что и в примеpe 2 , и делая то же отождествление, мы можем записать эти функции как $I_{k}\left(L^{+}+c_{-}\right)$ 
и $I_{l}\left(L^{-}+c_{+}\right)$, где $L-$ элемент общего положения из пространства $\mathfrak{g}^{*}$. В силу доказанной теоремы 3.3 они коммутируют по отношению к скобке $\{\cdot, \cdot\}_{c}$ на $\mathfrak{g}^{*}$ :

$$
\begin{aligned}
& \left\{I_{k}\left(L^{+}+c_{-}\right), I_{l}\left(L^{+}+c_{-}\right)\right\}_{c}=0, \\
& \left\{I_{k}\left(L^{-}+c_{+}\right), I_{l}\left(L^{-}+c_{+}\right)\right\}_{c}=0, \\
& \left\{I_{k}\left(L^{+}+c_{-}\right), I_{l}\left(L^{-}+c_{+}\right)\right\}_{c}=0,
\end{aligned}
$$

где сдвинутая скобка $\{\cdot, \cdot\}_{c}$ определена с помощью формулы (10).

\section{4. ИНТЕГРИРУЕМЫЕ ИЕРАРХИИ И ОТРИЦАТЕЛЬНЫЕ ПОТОКИ}

\section{1. Дубли, $R$-операторы и отрицательные потоки солитонных иерар-} хий. Используя результаты предыдущего раздела, можно вывести иерархию интегрируемых уравнений в частных производных, допускающих представления нулевой кривизны. Мы будем использовать один из ли-алгебраических подходов к теории солитонных уравнений [3]. Он базируется на том, что уравнения нулевой кривизны интерпретируются как условие согласованности двух вспомогательных уравнений Лакса на пространстве, дуальном к бесконечномерной алгебре Ли.

Для простоты мы сформулируем наш результат для градуированных бесконеч-

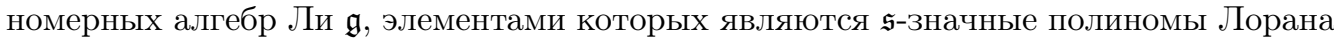
от одной комплексной переменной $\lambda$, или их подалгебр. Здесь $\mathfrak{s}-$ простая алгебра Ли в некоторой матричной реализации. Заметим, что в этом случае дуальное пространство $\mathfrak{g}^{*}$ может быть отождествлено с пространством формальных $\mathfrak{s - 3 н а ч н ы х ~}$ рядов Лорана.

Справедлива следующая теорема.

Теорема 4.1. Пусть $\mathfrak{g}$ - алгебра Ли, определенная выше. Обозначим через $\mathcal{G}$ ее дубль и через $\mathcal{G}^{*}$ - соответствующее дуалъное пространство. Пусть $J-$ идеал в алгебре $\mathcal{G}_{\mathcal{R}_{-}}$, имеющий конечную коразмерность. Обозначим через $\pi: \mathcal{G} \rightarrow \mathcal{G} / J$ естественную проекиию на фактор-алгебру, а через $\pi^{*}:(\mathcal{G} / J)^{*} \rightarrow \mathcal{G}^{*}$ - дуальное отображение. Если гамильтонианы $I_{k, \epsilon}\left(\pi^{*}(\mathcal{L})\right)$ на $\mathcal{G}^{*}$ являются конечными полиномами, то g-значные функиии $M_{k,+}=R_{+} \nabla I_{k, 1}\left(\pi^{*}(\mathcal{L})\right)$ и $M_{l,-}=R_{-} \nabla I_{l, 2}\left(\pi^{*}(\mathcal{L})\right)$ удовлетворяют уравнениям нулевой кривизны со значениями в алгебре $\mathfrak{g}$ :

$$
\begin{aligned}
& \frac{\partial M_{k, \pm}}{\partial t_{l}^{ \pm}}-\frac{\partial M_{l, \pm}}{\partial t_{k}^{ \pm}}+\left[M_{k, \pm}, M_{l, \pm}\right]=0, \\
& \frac{\partial M_{k, \pm}}{\partial t_{l}^{\mp}}-\frac{\partial M_{l, \mp}}{\partial t_{k}^{ \pm}}+\left[M_{k, \pm}, M_{l, \mp}\right]=0 .
\end{aligned}
$$

ДокАЗАТЕЛЬство. Заметим, что, используя коммутативность гамильтоновых потоков, порожденных гамильтонианами $I_{k, \epsilon}\left(\pi^{*}(\mathcal{L})\right), I_{l, \epsilon^{\prime}}\left(\pi^{*}(\mathcal{L})\right)$ (здесь $\left.\epsilon, \epsilon^{\prime} \in\{+,-\}\right)$, и два уравнения Эйлера-Арнольда (8), мы без труда можем вывести следующее уравнение:

$$
\operatorname{ad}_{\llbracket M_{k, \epsilon}, M_{l, \epsilon^{\prime}} \rrbracket} \pi^{*}(\mathcal{L})=0
$$

где

$$
\llbracket M_{k, \epsilon}, M_{l, \epsilon^{\prime}} \rrbracket \equiv \frac{\partial M_{k, \epsilon}}{\partial t_{l}^{\epsilon^{\prime}}}-\frac{\partial M_{l, \epsilon^{\prime}}}{\partial t_{k}^{\epsilon}}+\left[M_{k, \epsilon}, M_{l, \epsilon^{\prime}}\right]
$$


В рассматриваемом случае коприсоединенное представление алгебры $\mathfrak{g}$ (и, следовательно, ее дубля $\mathcal{G}$ ) может быть отождествлено с присоединенным, поэтому из уравнения (14) вытекает, что $\llbracket M_{k, \epsilon}, M_{l, \epsilon^{\prime}} \rrbracket$ принадлежит ядру оператора $\operatorname{ad}_{\pi^{*}(\mathcal{L})}$. Подстилающая конечномерная алгебра Ли s является полупростой, отсюда следует, что это ядро натянуто на выражение вида $\left(\pi^{*}(\mathcal{L})\right)^{k}$, где ассоциативные умножения в дубле понимаются как покомпонентные. Поскольку $J$ является идеалом конечной коразмерности в алгебре $\mathcal{G}_{\mathcal{R}_{-}}$, а также имеют место соотношения $\mathcal{G}_{\mathcal{R}}=\mathcal{G}_{\mathcal{R}_{+}} \ominus \mathcal{G}_{\mathcal{R}_{-}}$, $\mathcal{G}_{\mathcal{R}_{+}} \simeq \mathfrak{g}$, и в компонентной форме дуальное пространство $\mathcal{G}_{\mathcal{R}_{+}}^{*}$ может быть записано KaK

$$
\mathcal{R}_{+}^{*}(\mathcal{L})=\left(\begin{array}{c}
R_{+}^{*}\left(L_{1}+L_{2}\right) \\
-R_{-}^{*}\left(L_{1}+L_{2}\right)
\end{array}\right),
$$

мы получаем, что обе компоненты матрицы $\pi^{*}(\mathcal{L})$ и, следовательно, матриц $\left(\pi^{*}(\mathcal{L})\right)^{k}$ являются (каждая в своем направлении) полубесконечными формальными рядами Лорана. С другой стороны, функции $I_{k, \epsilon}\left(\pi^{*}(\mathcal{L})\right)$ и $I_{l, \epsilon^{\prime}}\left(\pi^{*}(\mathcal{L})\right)$ по условию теоремы представляют собой конечные полиномы. Следовательно, их матричные градиенты $M_{k, \epsilon}$ и $M_{l, \epsilon^{\prime}}$, а также $\llbracket M_{k, \epsilon}, M_{l, \epsilon^{\prime}} \rrbracket$ принадлежат алгебре $\mathfrak{g}$, т. е. алгебре $\mathfrak{s - з н а ч н ы х ~}$ полиномов Лорана (здесь $\mathfrak{g}$ реализована как диагональная подалгебра в $\mathfrak{g} \oplus \mathfrak{g})$. Сле-

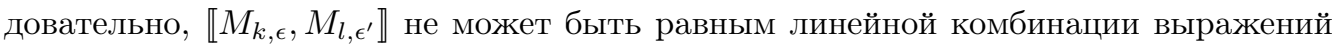
вида $\left(\pi^{*}(\mathcal{L})\right)^{k}$, кроме случая тривиальной линейной комбинации. Это означает, что $\llbracket M_{k, \epsilon}, M_{l, \epsilon^{\prime}} \rrbracket=0$. Теорема доказана.

ЗАмечАниЕ 5. Используя те же аргументы, можно показать, что данная теорема выполняется также и для более сложных бесконечномерных алгебр Ли, например для квазиградуированных алгебр Ли, которые отвечают за интегрируемость уравнений Ландау-Лившица и его различных обобщений [7], или для градуированных алгебр Ли типа $A_{\infty}, C_{\infty}, D_{\infty}$. В последнем случае вместо условия полиномиальности гамильтонианов нужно наложить менее жесткое условие.

ЗАмечАниЕ 6. Заметим, что уравнения (12), (13) определяют три типа интегрируемых иерархий: две "малые" иерархии, ассоциированные с алгебрами Ли $\mathfrak{g}_{R_{ \pm}}$ и определяемые уравнениями (12), и одну "большую" иерархию, ассоциированную со всей алгеброй Ли g и включающую оба типа уравнений (12) и (13). Уравнения (13) содержат $U-V$-пары с $U$-оператором, принимающим значение в алгебре $\mathfrak{g}_{R_{+}}$, и $V$-оператором, принимающим значение в алгебре $\mathfrak{g}_{R_{-}}$. Их можно интерпретировать как "отрицательные потоки” интегрируемых иерархий, ассоциированных с алгебрами $\mathfrak{g}_{R_{ \pm}}$.

4.2. Случай градуированных алгебр Ли. В этом пункте мы продемонстрируем, как описанная выше общая схема генерации $U-V$-пар, удовлетворяющих уравнению нулевой кривизны, работает для конкретных алгебр Ли. Мы сосредоточим наше внимание на простейшем из возможных случаев, связанным с градуированными алгебрами Ли.

4.2.1. Факторы дубля и инвариантные функции. Рассмотрим пример $\mathbb{Z}$-градуированных алгебр Ли и фактор-алгебр соответствующих дублей. По определению градуированных алгебр Ли мы имеем

$$
\mathfrak{g}=\sum_{j \in \mathbb{Z}} \mathfrak{g}_{j}, \quad\left[\mathfrak{g}_{i}, \mathfrak{g}_{j}\right] \subset \mathfrak{g}_{i+j}
$$


Используя свойства градуированности, легко получить разложение $\mathfrak{g}=\mathfrak{g}_{+}+\mathfrak{g}_{-}$, где $\mathfrak{g}_{+}=\sum_{j \geqslant 0} \mathfrak{g}_{j}$ и $\mathfrak{g}_{-}=\sum_{j<0} \mathfrak{g}_{j}-$ подалгебры Ли. Обозначим через $P_{ \pm}$операторы проецирования на подалгебры Ли $\mathfrak{g}_{ \pm}$. Тогда $R=P_{+}-P_{-}$есть классический $R$-оператор [17]. Стандартным образом [17] получаем, что $J_{+k}=\sum_{j>k} \mathfrak{g}_{j}$ и $J_{-l}=\sum_{j>l} \mathfrak{g}_{-l}-$ идеалы в подалгебах $\mathfrak{g}_{ \pm}$и в алгебре $\mathfrak{g}_{R}=\mathfrak{g}_{+} \ominus \mathfrak{g}_{-}$. Следовательно, можно рассмотреть фактор-алгебру $\mathfrak{g}_{R} /\left(J_{+k} \ominus J_{-l}\right)$ и фактор-алгебру $\mathcal{G}_{\mathcal{R}} /\left(J_{+k} \ominus J_{-l}\right)$ соответствующего дубля. Элементы последней фактор-алгебры имеют вид

$$
\left(\begin{array}{l}
X_{1}^{+}+X_{1}^{-^{\prime}} \\
X_{2}^{-}+X_{2}^{+^{\prime}}
\end{array}\right), \quad X_{1}^{+} \in \mathfrak{g}_{+}, \quad X_{1}^{-^{\prime}} \in \sum_{j=1}^{l} \mathfrak{g}_{-j}, \quad X_{2}^{-} \in \mathfrak{g}_{-}, \quad X_{2}^{+^{\prime}} \in \sum_{j=0}^{k} \mathfrak{g}_{j} .
$$

Соответствующие элементы дуального пространства имеют следующую явную формy:

$$
\mathcal{L}=\left(\begin{array}{l}
L_{1}^{+}+L_{1}^{-^{\prime}} \\
L_{2}^{-}+L_{2}^{+^{\prime}}
\end{array}\right), \quad L_{1}^{+} \in \mathfrak{g}_{+}^{*}, \quad L_{1}^{-^{\prime}} \in \sum_{j=1}^{l} \mathfrak{g}_{-j}^{*}, \quad L_{2}^{-} \in \mathfrak{g}_{-}^{*}, \quad L_{2}^{+^{\prime}} \in \sum_{j=0}^{k} \mathfrak{g}_{j}^{*}
$$

Отметим, что соответствующие компоненты оператора Лакса, а именно элементы $L_{1}=L_{1}^{+}+L_{1}^{-^{\prime}}$ и $L_{2}=L_{2}^{-}+{L_{2}^{+}}^{\prime}$, являются полубесконечными (т. е. бесконечными только в одном направлении).

Предположим, что на алгебре $\mathfrak{g}$ существует инвариантная билинейная форма $(\cdot, \cdot)$ такая, что $\left(\mathfrak{g}_{i}, \mathfrak{g}_{j}\right) \sim \delta_{i+j, 0}$. В этом случае можно отождествить пространства $\mathfrak{g}^{*}$ и $\mathfrak{g}$ и построить функцию Казимира второго порядка с помощью следующих формул:

$$
I_{2,1}^{0}=\frac{1}{2} \sum_{i \in \mathbb{Z}}\left(L_{1}^{(i)}, L_{1}^{(-i)}\right), \quad I_{2,2}^{0}=\frac{1}{2} \sum_{i \in \mathbb{Z}}\left(L_{2}^{(i)}, L_{2}^{(-i)}\right),
$$

где $L_{1,2}^{( \pm i)} \subset \mathfrak{g}_{\mp i}$. Отметим, что на указанных фактор-алгебрах приведенные выше выражения являются конечными полиномами, если пространство $\mathfrak{g}_{i}$ конечномерно.

Рассмотрим несколько примеров.

4.2.2. Общая неабелева система Тоды и градуированные алгебры Ли. Рассмотрим описанную выше конструкцию при $k=1, l=1$. В этом случае мы будем иметь

$$
I_{2,1}^{0}=\frac{1}{2}\left(L_{1}^{(0)}, L_{1}^{(0)}\right)+\left(L_{1}^{(1)}, L_{1}^{(-1)}\right), \quad I_{2,2}^{0}=\frac{1}{2}\left(L_{2}^{(0)}, L_{2}^{(0)}\right)+\left(L_{2}^{(1)}, L_{2}^{(-1)}\right),
$$

а матрица Лакса запишется следующим образом:

$$
\mathcal{L}=\left(\begin{array}{c}
L_{1}^{+}+L_{1}^{(-1)} \\
L_{2}^{-}+L_{2}^{(0)}+L_{2}^{(1)}
\end{array}\right), \quad L_{1}^{+}=\sum_{i=0}^{\infty} L_{1}^{(i)}, \quad L_{2}^{-}=\sum_{i=1}^{\infty} L_{1}^{(-i)} .
$$

Отметим, что $L_{1}^{(-1)}$ является центральным элементом благодаря тому факту, что $L_{1}^{(-1)} \in\left(\mathfrak{g}_{-} /\left[\mathfrak{g}_{-}, \mathfrak{g}_{-}\right]\right)^{*}$.

$M$-операторы, соответствующие интегралам $I_{2,1}^{0}, I_{2,2}^{0}$, имеют следующий вид:

$$
M_{2,1}^{0}=\mathcal{R}_{+} \nabla I_{2,1}^{0}=\left(\begin{array}{c}
\bar{L}_{1}^{(0)}+\bar{L}_{1}^{(1)} \\
\bar{L}_{1}^{(0)}+\bar{L}_{1}^{(1)}
\end{array}\right), \quad M_{2,2}^{0}=\mathcal{R}_{+} \nabla I_{2,2}^{0}=-\left(\begin{array}{c}
\bar{L}_{2}^{(-1)} \\
\bar{L}_{2}^{(-1)}
\end{array}\right)
$$


где

$$
\begin{gathered}
\bar{L}_{1}^{(0)}=\frac{1}{2} \nabla\left(L_{1}^{(0)}, L_{1}^{(0)}\right) \in \mathfrak{g}_{0}, \quad \bar{L}_{1}^{(1)}=P_{+} \nabla\left(L_{1}^{(1)}, L_{1}^{(-1)}\right) \in \mathfrak{g}_{1}, \\
\bar{L}_{2}^{(-1)}=P_{-} \nabla\left(L_{2}^{(1)}, L_{2}^{(-1)}\right) \in \mathfrak{g}_{-1} .
\end{gathered}
$$

Их компоненты, а именно операторы

$$
U=\bar{L}_{1}^{(0)}+\bar{L}_{1}^{(1)}, \quad V=\bar{L}_{2}^{(-1)},
$$

являются $U-V$-парами абелевых и неабелевых полей Тоды, как будет показано ниже.

Рассмотрим уравнение нулевой кривизны для данной $U-V$-пары:

$$
\frac{\partial U}{\partial t}-\frac{\partial V}{\partial x}+[U, V]=0
$$

Это уравнение дает следующие равенства для однородных компонент:

$$
\frac{\partial \bar{L}_{1}^{(1)}}{\partial t}=0, \quad \frac{\partial \bar{L}_{1}^{(0)}}{\partial t}=-\left[\bar{L}_{1}^{(1)}, \bar{L}_{2}^{(-1)}\right], \quad \frac{\partial \bar{L}_{2}^{(-1)}}{\partial x}=\left[\bar{L}_{1}^{(0)}, \bar{L}_{2}^{(-1)}\right] .
$$

Первое из этих уравнений удовлетворяется автоматически в силу того, что элемент $\bar{L}_{1}^{(1)}$ получен с помощью центрального элемента $L_{1}^{(-1)}$ и, следовательно, $\bar{L}_{1}^{(1)}=$ $C^{(1)}=$ const. Решим последнее из уравнений системы (16). Учитывая градуированность рассматриваемой алгебры, легко заметить, что $\mathfrak{g}_{0} \subset \mathfrak{g}$ является подалгеброй. Обозначим через $G_{0}$ соответствующую группу Ли. Пусть $g_{0} \in G_{0}$. Прямой проверкой легко убедиться, что замена переменных

$$
\bar{L}_{2}^{(-1)}=g_{0} C^{(-1)} g_{0}^{-1}, \quad \bar{L}_{1}^{(0)}=\left(\partial_{x} g_{0}\right) g_{0}^{-1}, \quad g_{0}=g_{0}(x, t),
$$

где $C^{(-1)}$ - постоянный элемент пространства $\mathfrak{g}_{-1}$, дает решение последнего из трех уравнений системы (16). Второе уравнение системы после подстановки решения третьего уравнения приобретает вид

$$
\partial_{t}\left(\left(\partial_{x} g_{0}\right) g_{0}^{-1}\right)=-\left[C^{(1)}, g_{0} C^{(-1)} g_{0}^{-1}\right]
$$

и представляет собой так называемое уравнение неабелевого поля Тоды [15].

4.3. Алгебры петель и стандартная цепочка Тоды. Основной пример изложенной выше конструкции связан с алгебрами петель. Пусть $\mathfrak{s}-$ простая алгебра Ли. Обозначим через $\mathfrak{g}=\mathfrak{s} \otimes \operatorname{Pol}\left(\lambda, \lambda^{-1}\right)$ соответствующую алгебру петель. Предположим, что $\mathfrak{s}$ снабжена автоморфизмом $\sigma: \mathfrak{s} \rightarrow \mathfrak{s}$ порядка $p$. Существует естественное разложение [18] $\mathfrak{s}=\sum_{i=0}^{p-1} \mathfrak{s}_{i}$ такое, что

$$
\mathfrak{s}_{k}=\left\{X \in \mathfrak{s} \mid \sigma(X)=e^{2 \pi i k / p} X\right\} .
$$

В частности, $\mathfrak{s}_{0}$ является подалгеброй, постоянной по отношению к действию автоморфизма $\sigma$.

Продолжим рассматриваемую градуировку на алгебру петель $\mathfrak{g}$, положив по определению

$$
\operatorname{deg} \lambda=p, \quad \operatorname{deg} X \otimes q(\lambda)=\operatorname{deg} X+\operatorname{deg} q(\lambda)
$$


В этом случае мы получим

$$
\mathfrak{g}_{j}=\left\{X(\lambda) \in \mathfrak{s} \otimes \operatorname{Pol}\left(\lambda, \lambda^{-1}\right) \mid \operatorname{deg} X(\lambda)=j\right\} .
$$

В частности, $\mathfrak{g}_{0}=\mathfrak{s}_{0}$. Соответствующая группа $G_{0} \subset G$ совпадает с группой Ли алгебры $\mathfrak{s}_{0}$. Уравнение (17) написано для элемента этой группы.

Рассмотрим наиболее интересный пример, отвечающий абелевой группе $G_{0}$. Пусть $\mathfrak{g}$ - алгебра петель в основной градуировке. Более детально, пусть $\sigma: \mathfrak{s} \rightarrow \mathfrak{s}-$ автоморфизм Кокстера. Обозначим через $h$ число Кокстера алгебры s. Мы имеем также соответствующую $\mathbb{Z}_{h}$-градуировку алгебры $\mathfrak{s}$ :

$$
\mathfrak{s}=\sum_{i=0}^{h-1} \mathfrak{s}_{i}
$$

Подалгебра $\mathfrak{s}_{0}$ совпадает с подалгеброй Картана $\mathfrak{h}=\operatorname{Span}_{\mathbb{C}}\left\{H_{\alpha_{i}} \mid i=1, \ldots, \operatorname{rank} \mathfrak{g}\right\}$, более того, $\mathfrak{s}_{k}=\operatorname{Span}_{\mathbb{C}}\left\{X_{\alpha}|\alpha \in \Delta,| \alpha \mid=k \bmod h\right\}$. Здесь $H_{\alpha_{i}}, X_{\alpha}$ - базис Картана-Вейля алгебры $\mathfrak{s}, \Delta$ - множество простых корней и $|\alpha|$ обозначает длину корня. В частности, $H_{\alpha_{i}}=\left[X_{\alpha_{i}}, X_{-\alpha_{i}}\right]$, если $\alpha_{i}$ - простые корни.

Опишем явно подпространство $\mathfrak{g}_{i}$. По определению имеем

$$
\mathfrak{g}_{0}=\mathfrak{h}, \quad \mathfrak{g}_{k}=\sum_{|\alpha|=k} \mathfrak{s}_{\alpha}+\lambda \sum_{|\alpha|=h-k} \mathfrak{s}_{-\alpha}, \quad k=1, \ldots, h-1,
$$

где $\mathfrak{s}_{\alpha}=\operatorname{Span}_{\mathbb{C}}\left\{X_{\alpha}\right\}$. Другие градуированные подпространства записываются следующим образом: $\mathfrak{g}_{k+n h}=\lambda^{n} \mathfrak{g}_{k}, k=1, \ldots, h-1$. Рассмотрим $U-V$-пару (15):

$$
U=\left(\partial_{x} g_{0}\right) g_{0}^{-1}+C^{(1)}, \quad V=g_{0} C^{(-1)} g_{0}^{-1} .
$$

В данном случае группа $G_{0}$ является абелевой и совпадает с подгруппой Картана, поэтому легко параметризировать ее элемент $g_{0}$ как $g_{0}=\exp \left\{\sum_{i=1}^{\operatorname{ranks}} \phi_{i} H_{\alpha_{i}}\right\}$ и получить, что

$$
\begin{aligned}
& U=\sum_{i=1}^{\text {rank } \mathfrak{s}} \partial_{x} \phi_{i} H_{\alpha_{i}}+\sum_{\alpha_{i} \in P} c_{\alpha_{i}}^{(1)} X_{\alpha_{i}}+\lambda c_{-\theta}^{(1)} X_{-\theta}, \\
& V=\sum_{\alpha_{i} \in P} c_{\alpha_{i}}^{(-1)} e^{-\alpha_{i}(\phi)} X_{-\alpha_{i}}+\lambda^{-1} c_{\theta}^{(-1)} e^{-\theta(\phi)} X_{\theta},
\end{aligned}
$$

где $P$ - множество простых корней, $\theta$ - старший корень и $H_{\alpha_{i}}$ - базисный элемент подалгебры Картана, соответствующий простому корню $\alpha_{i}$. В этой $U-V$-паре легко узнать $U-V$-пару конечнокомпонентного поля Тоды [14]. Соответствующие уравнения (17) принимают следующий вид:

$$
\partial_{t} \partial_{x} \phi_{i}=c_{\alpha_{i}}^{(1)} c_{-\alpha_{i}}^{(-1)} e^{-\alpha_{i}(\phi)}+a_{i} c_{-\theta}^{(1)} c_{\theta}^{(-1)} e^{\theta(\phi)}, \quad \phi=\sum_{i=1}^{\text {rank } \mathfrak{s}} \phi_{i} H_{\alpha_{i}},
$$

где константы $a_{i}$ определяются из разложения $H_{\theta}=\left[X_{\theta}, X_{-\theta}\right]$ :

$$
H_{\theta}=\sum_{i=1}^{\text {rank } \mathfrak{s}} a_{i} H_{\alpha_{i}}
$$


Из явной формы уравнений (18) легко видеть, что коэффициенты $c_{-\alpha_{i}}^{(-1)}, c_{\alpha_{i}}^{(1)}, c_{\theta}^{(-1)}$ и $c_{-\theta}^{(1)}$, если они не равны нулю, могут быть удалены перемасштабированием.

ЗАмЕчАНИЕ 7 . Используемый в настоящей работе $R$-оператор не является кососимметричным. Существует альтернативный подход к конечнокомпонентной двумерной системе Тоды, основанный на кососимметричном $R$-операторе [1], [19].

4.4. Бесконечнокомпонентная система Тоды. Пусть теперь $\mathfrak{g}=g l((\infty))$. Напомним, что это алгебра бесконечных матриц $M=\left(M_{i j}\right)_{i, j \in \mathbb{Z}}$, где $M_{i j}=0$ при $|i-j| \gg 1$. Она может быть рассмотрена как предел при $n \rightarrow \infty$ алгебры $\mathfrak{g}=g l(n)$, с которой мы имели дело в одном из приведенных выше примеров, однако $g l((\infty))$ нуждается в более аккуратных формулировках.

Базис в алгебре $g l((\infty))$ состоит из элементов $X_{i j}, i, j \in \mathbb{Z}$, со стандартными коммутационными соотношениями $\left[X_{i j}, X_{k l}\right]=\delta_{k j} X_{i l}-\delta_{i l} X_{k j}$. В терминах этого базиса определяются следующие градуированные подпространства в естественной $\mathbb{Z}$-градуировке: $\mathfrak{g}_{k}=\operatorname{Span}_{\mathbb{C}}\left\{X_{i j} \mid j-i=k\right\}$. На алгебре $g l((\infty))$ существует симметричная билинейная инвариантная форма $(\cdot, \cdot)$ такая, что $\left(X_{i j}, X_{k l}\right)=\delta_{k j} \delta_{i l}$. Используя эту форму, можно отождествить $\mathfrak{g}^{*}$ и $\mathfrak{g}$ так, что $\mathfrak{g}_{k}^{*}=\mathfrak{g}_{-k}$.

Рассмотрим классический дубль алгебры $g l((\infty))$. Применим конструкцию п. 4.2.1 к соответствующим факторпространствам по отношению к идеалам вида $J_{+k}$ и $J_{-l}$, где $k, l$ - фиксированные положительные целые числа. Элементы пространства, дуального к соответствующим фактор-алгебрам, имеют вид

$$
\mathcal{L}=\left(\begin{array}{l}
L_{1}^{+}+L_{1}^{-^{\prime}} \\
L_{2}^{-}+L_{2}^{+^{\prime}}
\end{array}\right) \in\left[\mathcal{G}_{\mathcal{R}} /\left(J_{+k} \ominus J_{-l}\right)\right]^{*},
$$

где

$$
\begin{array}{rlrl}
L_{1}^{+} & =\sum_{i=0}^{\infty} L_{1}^{(i)}, & L_{2}^{-} & =\sum_{i=1}^{\infty} L_{2}^{(-i)}, \\
L_{1}^{-^{\prime}}=\sum_{i=1}^{l} L_{1}^{(-i)}, & L_{2}^{+^{\prime}}=\sum_{i=0}^{k} L_{1}^{(i)}, & L_{s}^{(i)} \in g l((\infty))_{-i}, \quad s=1,2 .
\end{array}
$$

В частности, матрица Лакса $\mathcal{L}$ бесконечнокомпонентной системы Тоды соответствует $k=l=1$. В дальнейшем мы будем рассматривать только этот случай.

Обозначим естественный базис в пространстве, дуальном к алгебре $g l((\infty))$, теми же символами $X_{i j}$. В этом базисе оператор Лакса может быть задан через координатные функции $l_{1}^{(m)}(i), l_{2}^{(m)}(j)$ следующим образом:

$$
L_{s}^{(m)}=\sum_{i \in \mathbb{Z}} l_{s}^{(m)}(i-m) X_{i, i-m}, \quad s=1,2 .
$$

Как и раньше, координаты $l_{1}^{(-1)}(i)$ являются функциями Казимира скобки Ли-Пуассона на пространстве, дуальном к фактор-алгебре $\mathcal{G}_{\mathcal{R}} /\left(J_{+1} \ominus J_{-1}\right)$, поэтому их можно положить равными константам: $l_{1}^{(-1)}(i)=c_{i}$. Таким образом, $L_{1}^{(-1)}=\sum_{i \in \mathbb{Z}} c_{i} X_{i-1, i}$. Используя инвариантную форму $(\cdot, \cdot)$ на $g l((\infty))$, мы получаем два квадратичных 
гамильтониана $I_{2,1}^{0}, I_{2,2}^{0}$ на дубле алгебры $g l((\infty))$. Эти гамильтонианы имеют на рассматриваемом факторпространстве следующий вид:

$$
I_{2, s}^{0}=\frac{1}{2} \sum_{i \in \mathbb{Z}}\left(l_{s}^{(0)}(i)\right)^{2}+\sum_{i \in \mathbb{Z}} l_{s}^{(1)}(i) l_{s}^{(-1)}(i), \quad s=1,2 .
$$

Потоки, порождаемые данными гамильтонианами, записываются в форме Лакса:

$$
\frac{\partial \mathcal{L}}{\partial t_{s}}=\left[\widetilde{M}_{2, s}^{0}, \mathcal{L}\right], \quad s=1,2
$$

где $M$-операторы принимают значение в дубле алгебры $g l((\infty))$ и имеют следующий вид:

$$
\widetilde{M}_{2,1}^{0}=\mathcal{R}_{+} \widetilde{\nabla} I_{2,1}^{0}=\left(\begin{array}{c}
L_{1}^{(0)}+L_{1}^{(-1)} \\
L_{1}^{(0)}+L_{1}^{(-1)}
\end{array}\right), \quad \widetilde{M}_{2,2}^{0}=\mathcal{R}_{+} \widetilde{\nabla} I_{2,2}^{0}=\left(\begin{array}{c}
L_{2}^{(1)} \\
L_{2}^{(1)}
\end{array}\right)
$$

Несмотря на то что $\mathfrak{g}$ не является алгеброй петель, а функции $I_{2, s}^{0}$ не являются конечными полиномами, можно доказать аналогично теореме 4.1, что соответствующие $M$-операторы удовлетворяют уравнениям нулевой кривизны. Таким образом, можно записать следующую $g l((\infty))$-значную $U-V$-пару, удовлетворяющую уравнению нулевой кривизны:

$$
U=L_{1}^{(0)}+L_{1}^{(-1)}, \quad V=L_{2}^{(1)}, \quad L_{s}^{(i)} \in g l((\infty))_{-i}, \quad s=1,2 .
$$

Соответствующее уравнение нулевой кривизны дает уравнения

$$
\partial_{x} v_{i}=v_{i}\left(u_{i+1}-u_{i}\right), \quad \partial_{t} u_{i}=c_{i-1} v_{i-1}-c_{i} v_{i}, \quad i \in \mathbb{Z},
$$

где $u_{i} \equiv l_{1}^{(0)}(i), v_{i} \equiv l_{2}^{(1)}(i)$ и $x=t_{1}, t=t_{2}$. Замена переменных $u_{i}=\partial_{x} \phi_{i}$, $v_{i}=e^{\phi_{i+1}-\phi_{i}}$ сводит уравнения (19) к обычной бесконечнокомпонентной системе Тоды [11]

$$
\partial_{x t}^{2} \phi_{i}=c_{i-1} e^{\phi_{i}-\phi_{i-1}}-c_{i} e^{\phi_{i+1}-\phi_{i}}, \quad i \in \mathbb{Z} .
$$

Как и выше, константы $c_{i}$, если они не равны нулю, могут быть удалены перемасштабированием.

ЗАмечание 8. Уравнение (20) не совпадает по форме с уравнениями (18), отвечающими алгебре Ли $g l(n)$, потому что в этом пункте мы использовали другой базис в подалгебре Картана: базис $H_{i} \equiv X_{i i}$ вместо базиса $H_{\alpha_{i}}=X_{i i}-X_{i-1 i-1}$.

\section{5. Скобки Ли-Пуассона для бесконечнокомпонентной системы Тоды.} В этом пункте мы явно опишем $R$-операторную скобку Ли-Пуассона, которая соответствует естественному разложению $\mathrm{AKC}$, использованному в предыдущем пункте. Мы начнем со скобок Ли и затем учтем тот факт, что скобки Ли-Пуассона координатных функций повторяют скобки Ли базисных элементов алгебры.

Введем для удобства следующий базис в алгебре Ли $\mathfrak{g}=g l((\infty))$ :

$$
X^{(i)}(m) \equiv X_{m, i+m}, \quad i, m \in \mathbb{Z} .
$$

Коммутационные соотношения в этом базисе приобретают вид

$$
\left[X^{(i)}(m), X^{(j)}(n)\right]=\delta_{i+n-m, 0} X^{(i+j)}(m)-\delta_{m-n+j, 0} X^{(i+j)}(n),
$$


a $R$-оператор в этом случае записывается как $R=P_{+}-P_{-}$, где $P_{+}$и $P_{-}-$операторы, проецирующие на подалгебры Ли, которые натянуты соответственно на элементы $X^{(i)}(m)$ с $i \geqslant 0$ и $X^{(j)}(n)$ с $j<0$. При этом $R$-скобка на $g l((\infty))$ записывается следующим образом:

$$
\left[X^{(i)}(m), X^{(j)}(n)\right]_{R}=2(1-\sigma(i)-\sigma(j))\left(\delta_{i+n-m, 0} X^{(i+j)}(m)-\delta_{m-n+j, 0} X^{(i+j)}(n)\right),
$$

где $\sigma(i)=1$, если $i<0$, и $\sigma(i)=0$, если $i \geqslant 0$.

Для дубля алгебры $g l((\infty))$, т. е. для прямой суммы алгебр $g l((\infty)) \oplus g l((\infty))$, мы получаем следующую $R$-скобку, записанную для базисных элементов $X_{s}^{(i)}(m)$, $s=1,2$ :

$$
\begin{gathered}
{\left[X_{1}^{(i)}(m), X_{1}^{(j)}(n)\right]_{R}=2(1-\sigma(i)-\sigma(j))\left(\delta_{i+n-m, 0} X_{1}^{(i+j)}(m)-\delta_{m-n+j, 0} X_{1}^{(i+j)}(n)\right),} \\
{\left[X_{2}^{(i)}(m), X_{2}^{(j)}(n)\right]_{R}=2(\sigma(i)+\sigma(j)-1)\left(\delta_{i+n-m, 0} X_{2}^{(i+j)}(m)-\delta_{m-n+j, 0} X_{2}^{(i+j)}(n)\right),} \\
{\left[X_{1}^{(i)}(m), X_{2}^{(j)}(n)\right]_{R}=2(\sigma(i)-1)\left(\delta_{i+n-m, 0} X_{1}^{(i+j)}(m)-\delta_{m-n+j, 0} X_{1}^{(i+j)}(n)\right)+} \\
+2 \sigma(j)\left(\delta_{i+n-m, 0} X_{2}^{(i+j)}(m)-\delta_{m-n+j, 0} X_{2}^{(i+j)}(n)\right) .
\end{gathered}
$$

Скобки Ли-Пуассона для координатных функций $l_{s}^{(i)}(m)$ следуют из данных коммутационных соотношений:

$$
\begin{aligned}
&\left\{l_{1}^{(i)}(m), l_{1}^{(j)}(n)\right\}_{R}=2(1-\sigma(i)-\sigma(j))\left(\delta_{i+n-m, 0} l_{1}^{(i+j)}(m)-\delta_{m-n+j, 0} l_{1}^{(i+j)}(n)\right) \\
&\left\{l_{2}^{(i)}(m), l_{2}^{(j)}(n)\right\}_{R}=2(\sigma(i)+\sigma(j)-1)\left(\delta_{i+n-m, 0} l_{2}^{(i+j)}(m)-\delta_{m-n+j, 0} l_{2}^{(i+j)}(n)\right) \\
&\left\{l_{1}^{(i)}(m), l_{2}^{(j)}(n)\right\}_{R}=2(\sigma(i)-1)\left(\delta_{i+n-m, 0} l_{1}^{(i+j)}(m)-\delta_{m-n+j, 0} l_{1}^{(i+j)}(n)\right)+ \\
&+2 \sigma(j)\left(\delta_{i+n-m, 0} l_{2}^{(i+j)}(m)-\delta_{m-n+j, 0} l_{2}^{(i+j)}(n)\right) .
\end{aligned}
$$

Скобки Ли-Пуассона системы Тоды получаются с помощью следующей специализации: $l_{1}^{(-1)}(i)=c_{i}, l_{1}^{(k)}(i)=0$ для $k<-1$ и $l_{2}^{(j)}(i)=0$ для $j>1$. Эти скобки совпадают с первой пуассоновой структурой иерархии двумерного поля Тоды [16].

\section{5. КВАДРАТИЧНЫЕ И КУБИЧНЫЕ СТРУКТУРЫ НА ДУБЛЕ}

В этом разделе мы обсудим продолжение пуассоновых структур второго и третьего порядков с алгебры Ли $\mathfrak{g}$ на ее классический дубль $\mathcal{G}$ и согласованность соответствующих скобок.

5.1. Квадратичные пуассоновы структуры. Известно, что для некоторых классических $R$-операторов на $\mathfrak{g}$ можно определить, помимо линейной $R$-скобки, также скобку Пуассона второго порядка, которая играет важную роль в теории классических интегрируемых систем.

Далее мы предположим, что можно отождествить пространства $\mathfrak{g}$ и $\mathfrak{g}^{*}$ и, более того, алгебра $\mathfrak{g}$ обладает также структурой ассоциативной алгебры. Имеет место следующая теорема [20], [21].

ТЕОрема 5.1. Пусть классический $R$-оператор и его кососимметричная часть $\left(R-R^{*}\right) / 2$ удовлетворяют модифицированному классическому уравнению ЯнгаБакстера на алгебре $\mathfrak{g}$. Тогда: 
1) формула

$$
\left\{F_{1}, F_{2}\right\}_{2}=\left\langle L,\left[R\left(L \nabla F_{1}+\nabla F_{1} L\right), \nabla F_{2}\right]\right\rangle-\left\langle L,\left[R\left(L \nabla F_{2}+\nabla F_{2} L\right), \nabla F_{1}\right]\right\rangle
$$

определяет скобку Пуассона на пространстве $\mathfrak{g}$;

2) функиии Казимира алгебры $\mathfrak{g}$ коммутируют относительно скобок (21);

3) гамильтоновы уравнения движения по отношению к функции Казимира $I_{k}$ на $\mathfrak{g}$ и скобке (21) записъваются в форме Лакса:

$$
\frac{d L}{d t_{k}}=\left[R\left(L \nabla I_{k}+\nabla I_{k} L\right), L\right]
$$

4) скобки Пуассона (21) и (2) являются согласованными 2$)$.

Оказывается, что данная теорема может быть продолжена на дубль алгебры $\mathfrak{g}$.

Теорема 5.2. Пусть классический $R$-оператор и его кососимметричная часть $\left(R-R^{*}\right) / 2$ удовлетворяют модифииированному классическому уравнению ЯнгаБакстера на алгебре $\mathfrak{g}$. Тогда:

1) формула

$$
\left\{F_{1}(\mathcal{L}), F_{2}(\mathcal{L})\right\}_{2}=\left\langle\mathcal{L},\left[\mathcal{R}\left(\mathcal{L} \widetilde{\nabla} F_{1}+\widetilde{\nabla} F_{1} \mathcal{L}\right), \nabla F_{2}\right]\right\rangle-\left\langle\mathcal{L},\left[\mathcal{R}\left(\mathcal{L} \widetilde{\nabla} F_{2}+\widetilde{\nabla} F_{2} \mathcal{L}\right), \nabla F_{1}\right]\right\rangle
$$

задает скобку Пуассона на алгебре $\mathcal{G}$;

2) функиии Казимира алгебры $\mathcal{G}$ коммутируют относительно скобки Пуассона (22);

3) гамильтоновы уравнения движения по отношению к функиии Казимира $I_{k, \epsilon}$ на $\mathcal{G}$ и скобке (22) записываются в форме Лакса:

$$
\frac{d \mathcal{L}}{d t_{k}^{\epsilon}}=\left[R\left(\mathcal{L} \widetilde{\nabla} I_{k, \epsilon}+\widetilde{\nabla} I_{k, \epsilon} \mathcal{L}\right), \mathcal{L}\right], \quad \epsilon=1,2
$$

4) скобки Пуассона (22) и (3) являются согласованными.

ЗАмечАниЕ 9. Скобки Ли-Пуассона (22) могут быть записаны в терминах операторов $R, R_{ \pm}$в более явном виде:

$$
\begin{aligned}
& \left\{F_{1}\left(L_{1}, L_{2}\right), F_{2}\left(L_{1}, L_{2}\right)\right\}_{2}= \\
& =\left\langle L_{1},\left[R\left(L_{1} \nabla_{1} F_{1}+\nabla_{1} F_{1} L_{1}\right)-R_{-}\left(L_{2} \nabla_{2} F_{1}+\nabla_{2} F_{1} L_{2}\right), \nabla_{1} F_{2}\right]\right\rangle+ \\
& \quad+\left\langle L_{2},\left[R_{+}\left(L_{1} \nabla_{1} F_{1}+\nabla_{1} F_{1} L_{1}\right)-R\left(L_{2} \nabla_{2} F_{1}+\nabla_{2} F_{1} L_{2}\right), \nabla_{2} F_{2}\right]\right\rangle- \\
& \quad-\left\langle L_{1},\left[R\left(L_{1} \nabla_{1} F_{2}+\nabla_{1} F_{2} L_{1}\right)-R_{-}\left(L_{2} \nabla_{2} F_{2}+\nabla_{2} F_{2} L_{2}\right), \nabla_{1} F_{1}\right]\right\rangle- \\
& \quad-\left\langle L_{2},\left[R_{+}\left(L_{1} \nabla_{1} F_{2}+\nabla_{1} F_{2} L_{1}\right)-R\left(L_{2} \nabla_{2} F_{2}+\nabla_{2} F_{2} L_{2}\right), \nabla_{2} F_{1}\right]\right\rangle .
\end{aligned}
$$

ДокАЗАТЕЛЬСтво теоремы. Сначала отметим, что если $\mathfrak{g}$ является ассоциативной алгеброй, то $\mathcal{G}$ тоже является ассоциативной алгеброй и имеет естественную структуру прямой суммы ассоциативных алгебр. Чтобы доказать теорему, достаточно применить теорему 5.1 и доказать, что из условий теоремы 5.1 для

\footnotetext{
2) Напомним, что две скобки $\{\cdot, \cdot\}_{1}$ и $\{\cdot, \cdot\}_{2}$, заданные на одном и том же линейном пространстве, называются согласованными, если произвольная их линейная комбинация $a_{1}\{\cdot, \cdot\}_{1}+a_{2}\{\cdot, \cdot\}_{2}$ является скобкой Пуассона.
} 
$R$-оператора на $\mathfrak{g}$ следует, что классический $R$-оператор $\mathcal{R}$ и его кососимметричная часть $\left(\mathcal{R}-\mathcal{R}^{*}\right) / 2$ удовлетворяют модифицированному классическому уравнению Янга-Бакстера на дубле $\mathcal{G}$. Данное утверждение для оператора $\mathcal{R}$ следует автоматически из результатов работы [12]. Остается показать, что $\left(\mathcal{R}-\mathcal{R}^{*}\right) / 2$ удовлетворяет модифицированному классическому уравнению Янга-Бакстера на $\mathcal{G}$. Мы покажем это непосредственно. Имеем

$$
\mathcal{A} \equiv \frac{1}{2}\left(\mathcal{R}-\mathcal{R}^{*}\right)=\frac{1}{2}\left(\begin{array}{ll}
A & -S \\
S & -A
\end{array}\right), \quad A=R-R^{*}, \quad S=R+R^{*} .
$$

Подставляя это выражение в модифицированное классическое уравнение Янга-Бакстера, мы получаем, что оно эквивалентно следующим трем условиям:

$$
\begin{aligned}
A([A(X), Y]+[X, A(Y)])-[A(X), A(Y)] & =4[X, Y], \\
-S([S(X), Y])-A([X, S(Y)])+[A(X), S(Y)] & =0, \\
S([A(X), Y]+[X, A(Y)])-[S(X), S(Y)] & =0,
\end{aligned}
$$

которые должны быть выполнены для любых $X, Y \in \mathfrak{g}$. Первое из этих уравнений следует из условий теоремы. Используя определение операторов $A$ и $S$, легко показать, что (23) эквивалентны следующим трем уравнениям: для любых $X, Y \in \mathfrak{g}$

$$
\begin{aligned}
R([R(X), Y]+[X, R(Y)])-[R(X), R(Y)] & =[X, Y], \\
R^{*}\left(\left[R^{*}(X), Y\right]\right)-R^{*}([X, R(Y)])+\left[R^{*}(X), R(Y)\right] & =[X, Y], \\
R\left(\left[R^{*}(X), Y\right]+\left[X, R^{*}(Y)\right]\right)-\left[R^{*}(X), R^{*}(Y)\right] & =-[X, Y] .
\end{aligned}
$$

Уравнение (24а) - это модифицированное классическое уравнение Янга-Бакстера для оператора $R$. Уравнение (24б) выводится путем использования модифицированного классического уравнения Янга-Бакстера и условия существования невырожденной инвариантной формы на g. Наконец, уравнение (24в) следует из модифицированного классического уравнения Янга-Бакстера для оператора $A$. Теорема доказана.

ЗАмечАние 10. Теорема 5.2 означает, что квадратичная пуассонова структура, если она существует на алгебре $\mathfrak{g}$, всегда может быть продолжена на ее дубль $\mathcal{G}$. В частности, такое расширение существует для кососимметричных $R$-операторов на $\mathfrak{g}$, поскольку соответствующий $\mathcal{R}$-оператор на $\mathcal{G}$ тоже является кососимметричным.

5.2. Пуассонова структура третьего порядка. В этом пункте мы опишем кубическую пуассонову структуру на $\mathfrak{g}$ и ее продолжение на дубль $\mathcal{G}$. Мы будем использовать следующую теорему из работ [20], [21], которая справедлива при тех же предположениях об алгебре Ли $\mathfrak{g}$, что и в предыдущем пункте.

Tеорема 5.3. Пусть $R$ - классический $R$-оператор на алгебре g. Тогда:

1) формула

$$
\begin{aligned}
\left\{F_{1}(L), F_{2}(L)\right\}_{3}=\langle L & \left.,\left[R\left(L \nabla F_{1} L+L \nabla F_{1} L\right), \nabla F_{2}\right]\right\rangle- \\
& -\left\langle L,\left[R\left(L \nabla F_{2} L+L \nabla F_{2} L\right), \nabla F_{1}\right]\right\rangle
\end{aligned}
$$

определяет скобку Пуассона на пространстве $\mathfrak{g}$; 
2) функции Казимира алгебры $\mathfrak{g}$ коммутируют относительно скобок (25);

3) гамильтоновы уравнения движения по отношению к функции Казимира $I_{k}$ на $\mathfrak{g}$ и скобке (25) записъваются в форме Лакса:

$$
\frac{d L}{d t_{k}}=\left[R\left(L \nabla I_{k} L\right), L\right]
$$

4) если оператор $\left(R-R^{*}\right) / 2$ удовлетворяет модифицированному классическому уравнению Янга-Бакстера на алгебре $\mathfrak{g}$, то скобки Пуассона (25), (21) и (2) являются согласованными.

Аналогичное утверждение имеет место и для классического дубля алгебры $\mathfrak{g}$.

СлеДСтвИЕ 5.1. Пусть $R$ - классический $R$-оператор. Тогда:

1) формула

$$
\begin{aligned}
\left\{F_{1}(\mathcal{L}), F_{2}(\mathcal{L})\right\}_{3}=\langle\mathcal{L}, & {\left.\left[\mathcal{R}\left(\mathcal{L} \widetilde{\nabla} F_{1} \mathcal{L}+\mathcal{L} \widetilde{\nabla} F_{1} \mathcal{L}\right), \widetilde{\nabla} F_{2}\right]\right\rangle- } \\
& -\left\langle\mathcal{L},\left[\mathcal{R}\left(\mathcal{L} \widetilde{\nabla} F_{2} \mathcal{L}+\mathcal{L} \widetilde{\nabla} F_{2} \mathcal{L}\right), \widetilde{\nabla} F_{1}\right]\right\rangle
\end{aligned}
$$

определяет скобку Пуассона на пространстве $\mathcal{G}$;

2) функиии Казимира алгебры $\mathcal{G}$ коммутируют относителъно скобок (26);

3) гамильтоновы уравнения движения по отношению к функиии Казимира $I_{k, \epsilon}$ на $\mathcal{G}$ и скобке (22) записываются в форме Лакса:

$$
\frac{d \mathcal{L}}{d t_{k}^{\epsilon}}=\left[\mathcal{R}\left(\mathcal{L} \widetilde{\nabla} I_{k, \epsilon} \mathcal{L}\right), \mathcal{L}\right]
$$

4) если оператор $\left(R-R^{*}\right) / 2$ удовлетворяет модифицированному классическому уравнению Янга-Бакстера на алгебре $\mathfrak{g}$, то скобки Пуассона (26), (22) и (4) являются согласованными.

ЗАмечание 11. Скобка Ли-Пуассона (26) в терминах операторов $R, R_{ \pm}$может быть записана в более более явном виде:

$$
\begin{aligned}
& \left\{F_{1}\left(L_{1}, L_{2}\right), F_{2}\left(L_{1}, L_{2}\right)\right\}_{3}= \\
& =\left\langle L_{1},\left[R\left(L_{1} \nabla_{1} F_{1} L_{1}\right)-R_{-}\left(L_{2} \nabla_{2} F_{1} L_{2}\right), \nabla_{1} F_{2}\right]\right\rangle+ \\
& \quad+\left\langle L_{2},\left[R_{+}\left(L_{1} \nabla_{1} F_{1} L_{1}\right)-R\left(L_{2} \nabla_{2} F_{1} L_{2}\right), \nabla_{2} F_{2}\right]\right\rangle- \\
& \quad-\left\langle L_{1},\left[R\left(L_{1} \nabla_{1} F_{2} L_{1}\right)-R_{-}\left(L_{2} \nabla_{2} F_{2} L_{2}\right), \nabla_{1} F_{1}\right]\right\rangle- \\
& \quad-\left\langle L_{2},\left[R_{+}\left(L_{1} \nabla_{1} F_{2} L_{1}\right)-R\left(L_{2} \nabla_{2} F_{2} L_{2}\right), \nabla_{2} F_{1}\right]\right\rangle .
\end{aligned}
$$

ДокАЗАТЕЛЬСТво СЛЕДствия. Заметим, что, используя отождествление $\mathfrak{g}$ и $\mathfrak{g}^{*}$ как линейных пространств и как $\mathfrak{g}$-модулей, можно также отождествить пространства $\mathcal{G}$ и $\mathcal{G}^{*}$ как линейные пространства и $\mathcal{G}$-модули. Как было объяснено выше, дубль $\mathcal{G}$ ассоциативной алгебры $\mathfrak{g}$ всегда имеет структуру ассоциативной алгебры. Чтобы доказать следствие, осталось только применить теорему 5.3 к классическому дублю и принять во внимание, что, как было доказано в теореме 5.2 , антисимметричная часть оператора $\left(\mathcal{R}-\mathcal{R}^{*}\right) / 2$ удовлетворяет модифицированному классическому уравнению Янга-Бакстера на алгебре $\mathcal{G}$, если оператор $\left(R-R^{*}\right) / 2$ удовлетворяет модифицированному классическому уравнению Янга-Бакстера на алгебре $\mathfrak{g}$. 
ЗАмЕЧАНИЕ 12. Пуассоновы структуры второго и третьего порядков существуют и являются согласованными с линейной пуассоновой структурой (когда операторы $R$ и $\left(R-R^{*}\right) / 2$ удовлетворяют модифицированному классическому уравнению Янга-Бакстера). Они порождают коммутирующие гамильтоновы потоки, которые записываются в форме Лакса. Однако их использование в теории солитонов не является столь прямым и универсальным, как использование линейных скобок Пуассона. Действительно, для получения фазового пространства солитонных уравнений необходимо, как было объяснено выше, ограничивать динамику на некоторые линейные подпространства, совпадающие с факторпространствами соответствующей $R$-скобки. С другой стороны, эти линейные подпространства не являются, вообе говоря, факторпространствами по идеалам квадратичной и кубичной скобок. Чтобы ограничить эти скобки на соответствующие подпространства, необходимо применять дополнительно редукцию по Дираку. Для случая бесконечнокомпонентной системы Тоды, эта процедура была рассмотрена в работе [16].

В конце данного раздела объясним, почему существуют и являются согласованными с линейной структурой пуассоновы структуры второго и третьего порядков для случая бесконечнокомпонентной системы Тоды перед ограничением на факторпространства линейной скобки. Поскольку бесконечнокомпонентная система Тоды связана с дублем алгебры $g l((\infty))$, нам нужно доказать следующее утверждение.

УтВЕРЖДЕНИЕ 5.1. На дубле алгебры $g l((\infty))$, оснащенном $R$-оператором $A K C$ $R=P^{+}-P^{-}$, где $P^{+}$и $P^{-}-$oператорь, проецирующие на подалгебры верхнетреугольных и строго нижнетреугольных матрии, существуют квадратичная и кубичная пуассоновы структуры, согласованные с линейной скобкой Пуассона и въражаемые формулами (22) и (26) соответственно.

ДокАЗАтЕльство. Отметим, что дубль алгебры $g l((\infty))$ является, очевидо, ассоциативной алгеброй. Следовательно, чтобы применить теорему 5.2 и следствие 5.1 в рассматриваемом случае, достаточно доказать, что $\left(R-R^{*}\right) / 2$ удовлетворяет модифицированному классическому уравнению Янга-Бакстера на $g l((\infty))$. В нашем случае $R=P^{+}-P^{-}$, где $P^{+}$и $P^{-}-$операторы, проецирующие на подалгебры верхнетреугольных и строго нижнетреугольных матриц соответственно. Заметим также, что этот $R$-оператор может быть записан в виде $R=P_{+}+P_{0}-P_{-}$, где $P_{+}-$оператор, проецирующий на подалгебру Ли строго верхнетреугольных матриц, $P_{0}$ - оператор, проецирующий на подалгебру Ли диагональных матриц, и $P_{-} \equiv P^{-}$. Используя явную форму инвариантного спаривания, т. е. билинейную форму на алгебре $g l((\infty))$, легко показать, что $P_{ \pm}^{*}=P_{\mp}, P_{0}^{*}=P_{0}$ и, следовательно, $\left(R-R^{*}\right) / 2=P_{+}-P_{-}$. Из результатов работы [22] (см. также [9]) вытекает следующее утверждение. Если $\mathfrak{g}=\mathfrak{g}_{+}+\mathfrak{g}_{0}+\mathfrak{g}_{-}-$треугольное разложение алгебры Ли $\mathfrak{g}$, т. е. $\mathfrak{g}_{ \pm}, \mathfrak{g}_{0}-$ подалгебры Ли и $\mathfrak{g}_{0}$-модули, то любой оператор вида $P_{+}+R_{0}-P_{-}$, где $P_{ \pm}-$операторы, проецирующие на подалгебры $\mathfrak{g}_{ \pm}$, является решением модифицированного уравнения Янга-Бакстера на $\mathfrak{g}$ при условии, что $R_{0}$ - решение модифицированного уравнения Янга-Бакстера на $\mathfrak{g}_{0}$. Более того, если подалгебра $\mathfrak{g}_{0}$ абелева, то любой оператор $R_{0}$ включая тривиальный является решением модифицированного уравнения Янга-Бакстера на $\mathfrak{g}_{0}$. Поэтому оператор $P_{+}-P_{-}$является решением модифицированного уравнения Янга-Бакстера на $\mathfrak{g}$. 
С другой стороны, очевидно, что разложение алгебры $\mathfrak{g}=g l((\infty))$ в сумму строго верхнетреугольной, строго нижнетреугольной и диагональной частей, представляет собой треугольное разложение с абелевой (диагональной) частью $\mathfrak{g}_{0}$. Следовательно, рассмотренный $R$-оператор $\left(R-R^{*}\right) / 2=P_{+}-P_{-}$действительно является решением модифицированного уравнения Янга-Бакстера на алгебре $g l((\infty))$ и, в силу теоремы 5.2 и следствия 5.1, существуют квадратичные и кубичные пуассоновы структуры на алгебре $g l((\infty))$ и ее дубле. Утверждение доказано.

Благодарности. Т. В. Скрыпник выражает признательность Гвидо Карлету за обсуждение. Работа Б. А. Дубровина поддержана European Research Council (грант FroM-PDE) и Правительством РФ (мегагрант № 2010-220-01-077).

\section{Список литературы}

[1] Л. А. Тахтаджян, Л. Д. Фаддеев, Гамильтонов подход в теории солитонов, Наука, М., 1986.

[2] H. Flaschka, A. Newell, T. Ratiu, Physica D, 9:3 (1983), 300-323; 324-332.

[3] А. Ньюэл, Солитоны в математике и физике, Мир, М., 1989.

[4] H. Aratyn, J. F. Gomes, A. H. Zimerman, J. Phys. A, 39:5 (2006), 109.

[5] Z. Qiao, W. Strampp, Physica A, 313:3-4 (2002), 365-380.

[6] C.-L. Terng, K. Uhlenbeck, "Poisson actions and scattering theory for integrable systems", Surveys in Differential Geometry: Integral Systems, IV, eds. C.-L. Terng, K. Uhlenbeck, Internat. Press, Boston, MA, 1998, 315-402, arXiv: dg-ga/9707004.

[7] Т. В. Скрыпник, ТМФ, 142:2 (2005), 329-345.

[8] Т. В. Скрыпник, ТМФ, 155:1 (2008), 147-160.

[9] T. V. Skrypnyk, SIGMA, 4 (2008), 011, 19 pp.

[10] М. А. Семенов-Тян-Шанский, Функи. анализ и его прил., 17:4 (1983), 17-33.

[11] K. Ueno, K. Takasaki, Proc. Japan Acad. Ser. A Math. Sci., 59:5 (1983), 167-170; 59:6 (1983), 215-218.

[12] А. Рейман, М. А. Семенов-Тян-Шанский, Интегрируемые системы: теоретико-групповой подход, РХД, М., Ижевск, 2003.

[13] А. В. Михайлов, Писъма в ЖЭТФ, 30:7 (1979), 443-448.

[14] A. V. Mikhailov, M. Olshanetsky, A. Perelomov, Commun. Math. Phys., 79:4 (1981), $473-488$.

[15] Kh. Nirov, A. Razumov, Nucl. Phys. B, 782:3 (2007), 241-275.

[16] G. Carlet, Lett. Math. Phys., 71:3 (2005), 209-226.

[17] А. Рейман, М. А. Семенов-Тян-Шанский, “Теоретико-групповые методы в теории интегрируемых систем", Динамические системь-7, Современные проблемы математики. Фундаментальные направления, 16, ред. Р. В. Гамкрелидзе, ВИНИТИ, М., 1989, 119-193.

[18] В. Кац, Бесконечномерные алгебры Ли, Мир, М., 1993.

[19] P. P. Kulish, Lett. Math. Phys., 5:3 (1981), 191-197.

[20] W. Oevel, O. Ragnisco, Physica A, 161:1 (1989), 181-220.

[21] L.-C. Li, S. Parmentier, Commun. Math. Phys., 125 (1989), 545-563.

[22] F. Guil, M. Mañas, Lett. Math. Phys., 19 (1990), 89-95.

Поступила в редакцию 28.04.2011, после доработки 13.11.2011 